\title{
PERCHÉ È DIFFICILE COSTRUIRE \\ LE ISTITUZIONI
}

\author{
di Giovan Francesco Lanzara
}

\section{Il problema: costruire un'istituzione}

La costruzione di un'istituzione, qualsiasi cosa possa significare, è comunque un processo che implica la formazione di una struttura, o codice, o pattern regolato di comportamenti che acquista legittimità e «funziona» in un contesto sociale specifico, fino a diventare un elemento costitutivo della vita sociale, stabile e persistente nel corso del tempo. Tale struttura può essere in parte il risultato di un progetto deliberato, in parte l'esito non intenzionale dell'azione umana e dell'interazione sociale. Il più delle volte è congiuntamente l'uno e l'altro, in combinazioni variabili a seconda delle contingenze ${ }^{1}$. Se la struttura «funziona», essa potrà autosostenersi e riprodursi nel tempo. Ma in caso di «fallimento», provocato da un rendimento scadente o

La ricerca che ba dato origine a questo saggio è stata finanziata con fondi del Consiglio Nazionale delle Ricerche ed è stata svolta in parte presso l'Istituto Universitario Europeo di San Domenico di Fiesole e presso lo Stanford Center for Organizations Research, Stanford University. Ringrazio ambedue le istituzioni per essere state un ottimo ambiente di lavoro. Mi sento debitore in special modo nei confronti di Philipp Genschel e James March per le lunghe discussioni che mi hanno aiutato a sviluppare le mie idee. Ringrazio anche $i$ colleghi che banno contribuito al Seminario su Institution Building presso il Dipartimento di Organizzazione e Sistema Politico dell'Università di Bologna, e in particolare Giorgio Alberti, Claudio Ciborra, Giorgio Freddi, Carlo Guarnieri, Carlo Poni e Roberto Scazzieri per $i$ commenti critici alle precedenti versioni del saggio. Resto ovviamente il solo responsabile delle mie idee e dei miei errori.

1 «Costruire» è usato qui nel senso dell'inglese building o del tedesco bildung, ed evoca l'idea di azione e attività umana. Nessun dubbio che le istituzioni siano costrutti sociali, ma dire che l'azione collettiva crea istituzioni non implica necessariamente che esse siano progettate secondo un piano preordinato. Piuttosto, le istituzioni sono «entità emergenti» 0 «mondi» che spesso risultano dall'interazione complessa e dalla composizione di un gran numero di azioni componenti. In questa prospettiva, costruire un'istituzione significa sempre intervenire su un processo in corso, che può essere influenzato dall'azione ma non diretto in modo deterministico verso obbiettivi o stati finali fissati ex ante.

RIVISTA ITALIANA DI SCIENZA POLITICA / a. XXVII, n. 1, aprile 1997 
dall'erosione di legittimità morale e politica, non necessariamente verrà rimpiazzata in modo rapido e completo. Più verosimilmente le sue componenti fondamentali, anche se obsolete, continueranno a sopravvivere per lungo tempo «come frammenti di rotte catene, pendenti dalle volte di vecchi edifici» (Tocqueville 1969), senza alcuna funzione specifica, o forse subiranno un lento processo di mutamento, assumendo gradualmente nuove funzioni e significati col passare del tempo.

Nel corso della storia gli uomini hanno creato una grande varietà di istituzioni, ma raramente il processo di costruzione è avvenuto senza sforzo. Quando si cerca di imprimere alle pratiche sociali un ordine fondato su regole, o di governare le transazioni economiche e politiche, o di ricostruire un nuovo assetto istituzionale e politico dopo una crisi, si devono affrontare un gran numero di problemi. Le aspettative sono incerte, le preferenze variabili e ambigue, le identità confuse e instabili, le risorse scarse e non uniformemente distribuite, gli interessi molteplici e in conflitto tra loro. Aspettative, preferenze, identità e interessi sono incongruenti tra $\mathrm{i}$ diversi gruppi e tra $\mathrm{i}$ diversi orizzonti temporali, e inoltre cambiano in modo endogeno al processo di costruzione di istituzioni. La creazione di istituzioni è un compito difficile. Ma che cosa lo rende così difficile? In quali forme avviene, nonostante le difficoltà? E perché gli sforzi degli attori sociali sono spesso self-defeating?

In questo saggio cercherò di dare alcune risposte a questi interrogativi. Interpreterò la creazione di istituzioni come un problema di intelligenza adattiva e di apprendimento nell'allocazione e nell'uso di risorse materiali, cognitive, e simboliche nel tempo. Gli attori sociali e politici devono allocare le loro scarse risorse tra due fondamentali tipi di attività: l'esplorazione (exploration) di nuove configurazioni istituzionali o, alternativamente, lo sfruttamento (exploitation) e il perfezionamento di quelle già esistenti (March 1991). Exploration e exploitation sono due forme di apprendimento, entrambe necessarie alla costruzione di istituzioni. Ma il mantenimento di un equilibrio tra le due non è facile, non solo perché i trade-offs, in ogni fase del processo, non possono essere calcolati con precisione, ma anche perché, come vedremo, processi endogeni di apprendimento possono alternativamente condurre gli attori o a cicli di eccessiva exploration o a cicli di eccessiva exploitation, generando conseguenze controproducenti e mettendo in pericolo il bilanciamento tra l'una e l'altra attività (Levinthal e March 1993). In 
poche parole, i processi di costruzione di istituzioni possono essere autodistruttivi ${ }^{2}$.

Più che un'analisi comparativa del successo o del fallimento di eventi critici nei processi di institution building, vorrei qui sviluppare una prospettiva dinamica. Lo scopo di questo lavoro non è dunque l'individuazione di condizioni o fattori espliciti che determinano o facilitano la costruzione «non problematica» di istituzioni «buone», «efficienti», o «legittime», o che al contrario ne ostacolano la formazione. Ogni elenco finito di condizioni «fondamentali» o «strutturali» sembra destinato ad essere smentito dal verificarsi di contingenze inattese. Piuttosto che l'elaborazione di una macroteoria o la definizione di tipologie, la strategia analitica qui scelta è orientata alla descrizione di processi. Il mio interesse sta nell'individuazione dei micromeccanismi causali soggiacenti ai fenomeni osservati, meccanismi che possono essere rilevati in una grande varietà di processi sociali e politici ${ }^{3}$. Cercherò dunque di sviluppare un framework utile alla comprensione del processo di formazione di istituzioni e delle sue difficoltà a un certo livello di generalità, illustrando con esempi quando opportuno. L'astrazione, che forse qualcuno lamenterà, è per ora il prezzo necessario da pagare per non perdere il quadro d'insieme.

Complessità politica e complessità cognitiva. La recente letteratura ha per lo più concettualizzato la creazione di istituzioni come una soluzione al problema dell'azione collettiva (Olson 1966; Axelrod 1984; Taylor 1987; Elster 1989). Nelle loro ricerche gli analisti hanno esplorato quella che potremmo chiamare la complessità politica della creazione di istituzioni, di cui illustrazioni ormai divenute classiche sono la Tragedia dei Comuni (Hardin 1968) o il Dilemma del Prigioniero, situazioni decisionali tipiche esplorate a fondo dalla teoria dei giochi. La com-

2 Il modello della scelta razionale rappresenterebbe il problema della costruzione di istituzioni come il problema di trovare e di mantenere un equilibrio appropriato tra opportunità di investimento alternativo che generano benefici futuri. Ma questa è una rappresentazione inadeguata, perché le scelte sono complicate dal fatto che i benefici attesi di ciascuna opzione strutturale variano in dipendenza di fattori endogeni a loro volta variabili. In tali condizioni è difficile specificare quali siano i trade offs appropriati tra exploration e exploitation. La ricerca e la scelta razionale possono produrre conseguenze non desiderate. Una sequenza di scelte ottimali localmente o a breve termine possono condurre a distorsioni nel lungo termine.

3 Per una convincente difesa di questa strategia analitica nelle scienze sociali, alternativa allo sviluppo di macroteorie, si veda Elster (1993, capitolo primo). 
plessità politica è una funzione del numero degli attori rilevanti, del numero dei cleavages tra gli interessi, e della distanza tra $\mathrm{i}$ diversi scopi perseguiti dagli attori (Grandori 1995). La conflittualità degli interessi e le asimmetrie distributive rendono problematica la convergenza su soluzioni istituzionali stabili e condivise. Queste ultime sono sempre vulnerabili per la possibilità di defezione.

Una minore attenzione è stata invece dedicata, nell'ambito delle scienze politiche, alla complessità cognitiva o informativa, che costituisce un'altra cruciale fonte di difficoltà nella costruzione di istituzioni e, in generale, di complessi artefatti sociali come codici e sistemi di regole. La complessità cognitiva deriva non solo dall'incompletezza dell'informazione, dall'ambiguità e dalla debole strutturazione delle situazioni, dall'incertezza e dall'ignoranza di importanti relazioni causa/effetto o azioni/conseguenze, ma anche da interdipendenze sistemiche e intertemporali, da processi endogeni di apprendimento, da incoerenze strutturali e requisiti contraddittori che gravano sull'azione individuale e collettiva nel corso del tempo. La complessità cognitiva cresce con la distanza degli orizzonti temporali dei fini degli attori, rendendo in tal modo ancora più problematica la coerenza intertemporale e il bilanciamento dinamico tra attività di exploration e attività di exploitation.

La complessità cognitiva e la complessità politica interagiscono l'una con l'altra. La creazione di istituzioni avviene in un ambiente sociale e politico competitivo. Gli attori devono rispondere ad un ambiente che è costituito di altri attori che a loro volta rispondono alle scelte e alle azioni dei primi. Ciò che gli altri fanno in ogni fase temporale del processo dipende da ciò che ciascun specifico attore ha fatto o farà. Gli attori danno vita ad un'ecologia competitiva, in cui le azioni e le scelte di ciascuno diventano «ambiente» per l'altro, e in cui gli orizzonti temporali degli attori in competizione, tutti diversi, slittano continuamente (Schelling 1978 e 1980; March 1981; Levitt e March 1988). Per questi motivi la distribuzione presente e futura delle risorse e delle utilità attese ad esse associate dipende per ciascun attore dalle scelte degli altri attori e dagli orizzonti temporali di tali scelte. Ciò equivale a dire che il bilanciamento e la coerenza intertemporale tra exploration e exploitation sono interdipendenti, in ogni stadio del processo, con le soluzioni, circoscritte nello spazio e nel tempo, date di volta in volta al problema dell'azione collettiva (e anche con i fallimenti nel tro- 
vare tali soluzioni) ${ }^{4}$. L'allocazione degli sforzi tra exploration e exploitation richiede insomma sempre comparazioni sia intertemporali che tra i diversi gruppi di interesse.

Quantunque la dimensione politica e la dimensione cognitiva della costruzione di istituzioni siano profondamente compenetrate, in questo lavoro metterò la complessità politica, per una volta, sullo sfondo della mia argomentazione, e concentrerò invece l'attenzione sulla dimensione cognitiva e dell'apprendimento, specificatamente sulle molte versioni e implicazioni del dilemma tra exploration e exploitation ${ }^{5}$. La tesi che qui propongo è che, anche nel caso di completa cooperazione sociale, i tentativi di costruire istituzioni possono subire distorsioni o essere resi vani dalla complessità e dalle contraddizioni che emergono nella distribuzione intertemporale delle risorse. Nella prima parte cercherò di individuare le cause principali dei cicli di fallimento e dei dilemmi dell'azione. Nella seconda parte svilupperò implicazioni specifiche per quattro temi rilevanti: competenza, self-interest, identità, e fiducia. Nella terza e ultima parte esplorerò tre meccanismi fondamentali - punti focali, rendimenti crescenti, e bricolage - che possono contrastare la dinamica autodistruttiva e facilitare l'emergenza e il consolidamento di istituzioni. In particolare, si vedrà come il bricolage istituzionale possa combinare con successo l'attività di exploration con quella di exploitation.

\section{Origini e cicli dell'autodistruttività}

Un dilemma dell'apprendimento: exploration versus exploitation. Gli attori coinvolti in situazioni di crisi e di trasformazione istituzionale devono scegliere di impegnare le loro limitate risorse (attenzione, denaro, tempo, intelligenza, influenza) o nel-

4 A ben vedere, anche il dilemma exploration/exploitation può essere riformulato come un problema di azione collettiva, in quanto implica per ogni attore la distribuzione di risorse e il bilanciamento di interessi e utilità tra i diversi «sé» futuri dell'attore stesso lungo un asse temporale. Il «sé» attuale dell'attore gioca giochi strategici con i suoi «sé» futuri, non ancora conosciuti e fondamentalmente impredicibili (Pizzorno 1986a).

5 I fattori di complessità politica verranno evocati però ogni volta che le diverse preferenze e identità in conflitto complicano il problema dell'allocazione intertemporale delle risorse, ostacolando la formazione di una «massa critica» nel riconoscimento reciproco e nel coordinamento. 
l'esplorazione di assetti istituzionali alternativi oppure nello sfruttamento e nell'utilizzazione di quelli già esistenti. L'esplorazione di nuovi assetti richiede che gli attori orientino la gran parte dei loro sforzi all'indagine e alla ricerca attiva. Implica sperimentazione, propensione al rischio, invenzione, attitudini al gioco e alla scoperta, diversità, e disponibilità a convivere con l'ambiguità. L'exploration estende «l'ombra del futuro» (Axelrod 1984). L'exploitation, al contrario, si caratterizza per il raffinamento e il perfezionamento delle routine e degli assetti già disponibili, l'avversione al rischio, la scelta e l'efficienza selettiva, vale a dire per un atteggiamento più conservatore nell'allocazione degli sforzi - la dominanza del «tenace passato» (Kuran 1988). Nell'exploitation «l'ombra del passato» oscura «'immagine del futuro».

L'exploration genera variabilità, mentre l'exploitation garantisce affidabilità. Il dilemma nasce perché variabilità e affidabilità sono entrambe necessarie, sono entrambe valutate nella stessa misura dagli attori, e hanno entrambe conseguenze positive e negative. Gli sforzi orientati all'esplorazione ad esclusione dello sfruttamento tendono a generare situazioni in cui i costi della sperimentazione sono elevati, inevitabili, e sostenuti nel breve periodo, mentre $\mathrm{i}$ benefici ottenuti possono essere minimi, incerti, e distanti nel tempo. Una tale situazione richiede una elevata capacità di sostenere il rischio e la pazienza di attendere risultati che arriveranno eventualmente nel lungo periodo. $\mathrm{Al}$ contrario, gli sforzi dedicati all'exploitation ad esclusione dell'esplorazione tendono a riprodurre e a rinforzare assetti istituzionali subottimali o disfunzionali. A loro vantaggio però, le attività di exploitation ricevono un feedback più rapido, presentano minori rischi, e i benefici sono più a breve termine.

Feedback rapido e feedback lento. Un tema ricorrente nella costruzione di istituzioni è la disparità tra le considerazioni a breve e a lungo termine. La crisi di un regime politico accresce la salienza psicologica della sopravvivenza nel breve periodo, ma rende tale sopravvivenza ancora più problematica. Gli orizzonti temporali della decisione e dell'azione tendono ad accorciarsi. Gli attori hanno aspettative a breve e si aspettano un feedback positivo rapido dalle loro azioni. Questo è necessario per sostenere l'azione e far partire il processo di costruzione. La mancanza di un rapido rinforzo positivo deprime ulteriormente le aspettative e inibisce l'azione. Ma il consolida- 
mento di nuovi assetti istituzionali richiede generalmente investimenti che producono rendimenti nel lungo periodo e hanno un feedback lento, dilazionato nel tempo. L'emergenza di complesse strutture di coordinamento è spesso un lento processo evolutivo che necessita di passi intermedi e il sostegno di «impalcature» componenti che devono essere a loro volta costruite. La generazione di opzioni multiple e l'esplorazione della loro realizzabilità e delle loro conseguenze richiedono tempo. Il feedback d'informazione è lento e ambiguo. Inoltre, opzioni alternative per la decisione e l'azione si presentano in sequenza temporale. I rendimenti degli investimenti sono incerti e spesso ritardati nel tempo. Per tali motivi, se gli attori nelle loro attività danno la priorità a guadagni rapidi e nel breve periodo, rischiano di creare condizioni che inibiscono gli impegni e gli investimenti nel lungo periodo, rendono impossibile la ricerca di soluzioni durevoli, e rinviano a tempo indeterminato ogni serio tentativo di edificare istituzioni. D'altra parte, se si impegnano in un'impresa di lungo periodo, rischiano nel breve di non avere le risorse sufficienti per sostenere i loro sforzi e le loro aspettative per un così lungo arco di tempo, e il processo di costruzione istituzionale si arresta. La risoluzione di questo dilemma non è un compito facile. Le cose si complicano perché attori diversi hanno non solo diverse aspettative, ma anche diversi orizzonti temporali per le loro aspettative: mentre alcuni possiedono un più elevato grado di tolleranza dell'ambiguità e si impegnano in attività di esplorazione, altri non la possiedono e pretendono risultati e benefici immediati. Il coordinamento dei molteplici orizzonti temporali degli attori è allo stesso tempo sia un elemento fondamentale che un prodotto del processo di costruzione di istituzioni: l'uno non può essere conseguito senza l'altro, e può essere conseguito solo attraverso l'altro.

Costi immersi. Gli assetti istituzionali e organizzativi esistenti (regole, codici, strutture, insieme con i modelli d'azione, le competenze specializzate e le rappresentazioni ad esse associate) incorporano «costi immersi» (sunk costs) per la società, cioè costi sostenuti per la creazione originaria di risorse produttive che non possono essere facilmente recuperate (Stinchcombe 1968). I costi immersi hanno la loro origine in un capitale di conoscenza, fiducia, aspettative condivise e obbligazioni reciproche, che è stato cristallizzato in una configurazione struttu- 
rale fissa ${ }^{6}$. Più lunga è stata la vita di un'istituzione e maggiori i suoi benefici passati, maggiori saranno plausibilmente $i$ costi immersi. I costi sostenuti per l'eventuale restaurazione delle istituzioni esistenti si sommano ai costi immersi originari. Nella costruzione di istituzioni dunque, in quale misura gli attori devono dedicare le loro energie e risorse alla conservazione o restaurazione dell'assetto istituzionale esistente, ancorché degradato cercando di salvare il salvabile -, o al contrario devono concentrare i loro sforzi nell'esplorazione di nuove possibili configurazioni? Sorgono problemi sia che si scelga l'uno o l'altro «corno» del dilemma: le alternative disponibili non sono scelte indipendenti, e le loro conseguenze possono generare problemi ancora più gravi. Da un lato, se gli attori scelgono di restare ancorati all'assetto istituzionale esistente e di cercare di migliorarlo, in primo luogo potrebbero commettere l'errore di consumare risorse per ciò ch'essi potrebbero presto scoprire essere una struttura irreversibilmente degradata, sostanzialmente irrecuperabile; in secondo luogo potrebbero creare condizioni che riducono ulteriormente la loro capacità e disponibilità a cambiare la struttura quando il cambiamento diventa ancora più necessario e non può più essere rinviato. Dall'altro lato, se gli attori passano a costruire un assetto istituzionale ex novo, in primo luogo potrebbero fare l'errore di gettar via risorse che, pur depauperate, potrebbero ancora in parte essere sfruttate; in secondo luogo potrebbero dedicare risorse e energie a ciò che potrebbe più tardi rivelarsi una soluzione troppo prematura, dunque intrinsecamente instabile, intenibile e a vita breve, generando così il bisogno di una ulteriore ricerca. Le conseguenze controintuitive e non volute di un «salto» troppo avventuroso sono instabilità, ulteriore ambiguità, e a volte il ripiegamento su posizioni più conservatrici.

Il riorientamento dell'attenzione e delle risorse dai vecchi ai nuovi assetti dipende dai rischi percepiti e dalle incertezze che nascono quando si abbandonano sentieri familiari per percorsi sconosciuti. Creando patb-dependency e rendimenti crescenti (David 1986; Arthur 1988 e 1989; David 1992), i costi immersi

6 I sunk costs non sono equamente distribuiti tra i gruppi sociali. Esistono asimmetrie e disuguaglianze che influenzano la disponibilità dei diversi attori a conservare o a smantellare specifici assetti istituzionali. Questo rende le cose più complicate, ma per il momento, per condurre in porto la presente trattazione, sospendiamo tale fattore di complicazione. 
tendono a preservare strutture e patterns d'azione tradizionali. Accontentarsi dell'assetto esistente per non voler incorrere nei costi e nei rischi del cambiamento può creare condizioni che rendono un futuro cambiamento più necessario e al tempo stesso più laborioso, e conducono a costi di cambiamento ancor più elevati. Ma assumersi il rischio di saltare prematuramente a una nuova configurazione per non voler sostenere le esternalità negative e i costi di mantenimento/restaurazione della vecchia struttura può creare condizioni d'instabilità e di insicurezza che dopo un certo tempo spingono gli attori a ristabilire molti degli assetti e delle regole preesistenti. Così, sia l'avversione che la propensione al rischio producono conseguenze non volute. Sia l'exploration che l'exploitation possono rivelarsi autodistruttive.

Cicli di fallimento. Sia l'exploration che l'exploitation sono affette da cicli autodistruttivi, che possono assumere la forma di «ricerche a tentoni», «blocchi», o brusche inversioni di tendenza.

\section{a) Ricerche a tentoni: eccessiva instabilità}

Dopo una crisi istituzionale o durante una transizione politica, le identità, le preferenze e le aspettative tendono alla variabilità e diventano estremamente sensibili alle contingenze. Vengono cercati nuovi assetti, ai quali però non si dà il tempo di consolidarsi. Nuovi esperimenti istituzionali vengono interrotti prima che abbiano la possibilità di produrre un qualche beneficio. È improbabile d'altra parte che nelle fasi iniziali della loro vita i nuovi assetti istituzionali abbiano rendimenti elevati. Questi si manifestano in genere nel lungo periodo, come effetto dell'accumulazione di esperienza. Accade così che i nuovi assetti vengano prematuramente giudicati inadeguati e insoddisfacenti, vengano messi da parte, e che parta un altro ciclo di sperimentazione parziale. La conseguenza di ciò è che diventa difficile mettere seriamente alla prova e stabilizzare definitivamente una nuova struttura. Una sequenza di esplorazioni incomplete o che non hanno dato esiti positivi può facilmente essere descritta come una storia di fallimenti e delusioni piuttosto che come una storia di ricerca e sperimentazioni.

Un'ulteriore spinta all'instabilità è costituita dal fatto che nel corso del processo di esplorazione emergono, in seguito a scelte fatte precedentemente o per lo slittamento endogeno del- 
le preferenze, nuove alternative che si presentano come attraenti e che spingono a nuove esplorazioni. Specialmente dopo una grave crisi politica e durante il successivo periodo di transizione può svilupparsi un'eccessiva propensione a rivedere o a rinegoziare decisioni prese in precedenza. Se questo è il caso, la formazione di regole, identità e preferenze stabili viene ostacolata, e non è possibile apprendere dall'esperienza ${ }^{7}$. Il fenomeno potrebbe essere chiamato la «sindrome dell'esplorazione»: plus ça change et... plus ça change. L'esplorazione diventa una ricerca a tentoni (random walk), una sorta di avventura senza fine. La via intrapresa non porta da nessuna parte, o porta ad una situazione in cui subentrano stanchezza e intolleranza per l'eccessivo livello d'incertezza. L'esplorazione può allora volgersi in un'attività autodistruttiva perché l'orientamento a obbiettivi di lungo periodo crea nel breve condizioni che possono vanificare la materializzazione del lungo periodo.

\section{b) «Blocchi»: eccessiva stabilità}

L'exploitation con esiti positivi di un dato assetto istituzionale può condurre ad un ulteriore perfezionamento dello stesso assetto. Questa è la ben nota trappola del successo (Levinthal e March 1993). L'accumulazione di esperienza in uno specifico ambito di attività accresce la competenza in quello specifico ambito, generando rendimenti crescenti e un maggiore impegno in quell'attività. $\mathrm{Ma}$ il ciclo genera anche conseguenze non volute: se da un lato esso produce ritorni positivi nel breve termine, dall'altro tende a conservare e a rafforzare frameworks sempre più specializzati. In questo caso la stabilità genera ulteriore stabilità fino al punto in cui un'eccessiva stabilità può diventare una trappola. Strutture, routine e competenze si radicano così profondamente da diventare resistenti al cambiamento. Le modifiche incrementali al margine contribuiscono solo ad accrescere la persistenza nel tempo delle istituzioni. Questa potrebbe essere chiamata la «sindrome dell'exploitation»: plus ça change

7 Le ricerche di Zhou (1993) hanno mostrato che: a) la velocità di cambiamento delle regole aumenta con il numero cumulativo dei cambiamenti precedenti, e che: $b$ ) la velocità di cambiamento di una regola diminuisce con la sua età. In altri termini, quando alla regola viene concesso un tempo sufficiente per vivere ed essere utilizzata, essa acquista legittimità e genera rendimenti crescenti, in tal modo «mettendo radici». Al contrario, cambiamenti troppo frequenti delle regole generano una spinta alla produzione di ulteriori regole. 
et... plus c'est la même chose, qualcosa di molto simile alla famosa sindrome del Gattopardo ${ }^{8}$.

Una storia di ripetuti ed efficaci «sfruttamenti» di un assetto istituzionale tende a creare capacità specifiche e a rinforzare identità specifiche, che sono poco adatte all'esplorazione. Man mano che si procede nell'exploitation di uno specifico assetto istituzionale il costo per passare all'esplorazione aumenta. Cadiamo così nella trappola della competenza. Quando la competenza con le procedure e le routine esistenti migliora, la sperimentazione di altre diventa meno attraente. Si tende in genere a fare ciò che si sa far meglio, ma in tal modo è probabile che si riduca la capacità di cercare possibili alternative. Per queste ragioni, una storia di successivi perfezionamenti tende a inibire le abilità e le attitudini appropriate a intraprendere attività esplorative. Consolidando strutture, routine e competenze già esistenti, l'exploitation distrugge variabilità e genera rigidità. Essa incorpora una dinamica potenzialmente autodistruttiva in quanto può portare al perfezionamento di istituzioni inefficienti o con un deficit di legittimità, le quali diventano più affidabili nel breve termine ma più vulnerabili nel lungo periodo.

\section{c) Rapide inversioni di preferenze}

Non è detto che l'eccessiva instabilità causata da una vana esplorazione di configurazioni istituzionali alternative e l'eccessiva stabilità causata dal perfezionamento della stessa struttura siano sempre e necessariamente processi che si autorinforzano. Entrambe possono essere interrotte da forze endogene contrastanti. Condizioni di prolungata instabilità e variabilità possono provocare un mutamento endogeno di preferenze dall'exploration all'exploitation. L'esperienza di ripetuti fallimenti a consolidare una struttura può condurre gli attori a ridurre la propensione al rischio. Non si possono sopportare a lungo elevati livelli di incertezza. Il livello delle aspettative si abbassa rapidamente (March 1988; Lant 1992). Se l'esplorazione non dà i risultati attesi, un modo facile e diretto per ridurre l'incertezza e l'insicurezza sociale consiste nel tornare ad assetti e routine già sperimentati. I vecchi copioni e modelli di comportamento vengono allora riattivati.

8 «Se vogliamo che tutto rimanga com'è bisogna che tutto cambi» (Tomasi di Lampedusa 1958). 
Quando l'instabilità e l'incertezza percepita diventano intollerabili per gli attori, essi rinunciano all'esplorazione e ristabiliscono molti dei precedenti assetti, che sono più familiari. Questi sono più sicuri e prontamente disponibili. La sicurezza viene preferita alla variabilità e alla diversità. Dopo un periodo di esplorazione, se non si ottengono risultati significativi, si tende a diventare più conservatori e ad autoimporsi limitazioni e vincoli; o semplicemente si diventa indifferenti alle novità. Per esempio, un forte impegno iniziale verso una riforma sociale o una nuova policy può gradualmente cadere nel momento in cui se ne devono pagare $i$ costi. Le priorità vengono invertite. Le coalizioni politiche possono smembrarsi quando gli effetti di una politica cominciano a manifestarsi. Le identità collettive evocate da una mobilitazione sociale o dai media possono frantumarsi in identità locali in competizione tra loro, diventando volatili e vulnerabili.

Quando invece l'eccessivo sfruttamento o l'obsolescenza di uno specifico assetto istituzionale riducono il suo rendimento, può aver luogo un'inversione nella direzione opposta, dall'exploitation all'exploration. In questo caso si verifica una violazione della condizione di stabilità causata da un cambiamento endogeno delle preferenze. La stabilità, la sicurezza nel breve, e i facili guadagni perdono valore rispetto alla propensione al rischio e all'orientamento al lungo termine. Il livello delle aspettative si innalza. Risorse e attenzione vengono riorientate alla ricerca e all'invenzione di nuove routine e strutture. L'interesse alla variabilità e alla diversità prende il sopravvento sull'interesse alla sicurezza. Lo status quo può divenire intollerabile, almeno per alcuni gruppi sociali, come accade comunemente nella crisi o nel crollo di un regime politico.

\section{Istanze e implicazioni della dinamica autodistruttiva}

Nelle pagine seguenti esaminerò brevemente alcune istanze e implicazioni di tale dinamica nella creazione di istituzioni, concentrandomi su quattro problemi specifici che mi sembrano rilevanti: risorse e competenze, self-interest, identità, e fiducia.

Competenze e risorse. Quando un regime politico crolla, nasce un problema di competenza. Le istituzioni tendono a generare e a sviluppare risorse e competenze che sono specifiche- 
per-quelle-istituzioni e che diventano «dotazioni» di specifici attori. Queste competenze integrano le esperienze accumulate nel tempo, vale a dire ciò che gli attori hanno imparato a fare con una specifica struttura di regole o all'interno di uno specifico assetto istituzionale: esse sono incorporate sia in abilità pratiche e conoscenze individuali che in routine sociali e amministrative praticate collettivamente. Tali competenze specifiche sono essenziali al sostegno e alla riproduzione delle istituzioni. In assenza di una simile base di competenza, istituzioni come le burocrazie governative, le agenzie pubbliche, gli organi politici, le imprese, e gli istituti culturali non potrebbero funzionare efficacemente.

Nel corso dello sviluppo e consolidamento di una determinata configurazione istituzionale gli attori apprendono abilità, routine e pratiche specifiche che sono profondamente compenetrate con quella configurazione: esse vengono formate all'interno e per mezzo della configurazione, che dà loro valore e significato e nell'ambito della quale esse «funzionano» efficacemente. Ad esempio, gli attori apprendono codici, sviluppano conoscenze, costruiscono reticoli sociali di relazioni interpersonali (Granovetter 1985 e 1990), o realizzano infrastrutture materiali, che sono tutti elementi specifici per un determinato assetto istituzionale. Questi effetti prodotti dal learning-by-using effetti largamente endogeni - costituiscono potenti meccanismi di rinforzo per la stabilità istituzionale, perché generano rendimenti crescenti nell'adozione di specifiche soluzioni istituzionali (David 1986; Arthur 1988; Jepperson 1991).

Quando un vecchio regime crolla e un nuovo regime deve essere istituito, la creazione di nuove istituzioni è condizionata dal repertorio esistente di competenze e risorse. Il più delle volte queste dotazioni sono distribuite nella popolazione in modo non equilibrato: in genere esse sono appannaggio quasi esclusivo di una élite politica o sociale, della classe dirigente, o comunque dei gruppi che hanno un elevato interesse alla sopravvivenza dell'ancien régime. Ciò che può essere realisticamente realizzato nella fase «nascente» della costruzione di un nuovo assetto istituzionale dipende dalle risorse e dalle competenze degli individui e dei gruppi che erano strettamente allineati o legati alle vecchie istituzioni. Riforme politiche innovative o assetti istituzionali progettati da innovatori sociali e politici spesso non possono essere implementati perché per attuare $i$ nuovi progetti si deve fare affidamento sul personale dirigente del 
vecchio regime. Questi burocrati - banchieri, quadri politici, dirigenti pubblici e privati, funzionari, e ogni tipo di personale amministrativo di ogni ordine e grado non sono risorse facilmente sostituibili: essi possiedono la conoscenza specifica della macchina amministrativa e un'esperienza pratica che può essere acquisita solo attraverso un lungo e costoso processo di apprendimento (learning-by-doing). Invero, anche i più violenti shocks istituzionali a mala pena toccano i quadri amministrativi e governativi intermedi. D'altra parte, anche quando si verifica una consistente importazione di competenze esterne dai settori specifici dell'economia e del management, dalla società civile, o dal mondo accademico, passa del tempo prima ch'esse funzionino efficacemente e che diventino produttive nel nuovo ambiente politico.

Ciò rende la creazione di nuove istituzioni difficile e dilemmatica: se le competenze e le risorse esistenti vengono tenute fuori e non impiegate nel processo d'innovazione, la costruzione di istituzioni risente della mancanza di personale esperto, e procede zoppicando; al contrario, se le competenze esistenti vengono utilizzate, esse tendono ad applicare molte delle vecchie regole, pratiche e abitudini, che possono sabotare ogni tentativo d'innovazione e riattivare molte delle caratteristiche del regime precedente (specifici individui, élites, istituzioni). Dopo un certo lasso di tempo gli esiti del processo di trasformazione cominciano ad assomigliare in modo sinistro proprio a ciò di cui gli innovatori volevano sbarazzarsi. Il bisogno di expertise politica e amministrativa può condurre i nuovi regimi a riportare in vita i vecchi. $\mathrm{Ma}$ così essi rischiano di perdere la superiorità morale in base alla quale essi invocano riconoscimento e legittimità, ossia perdono il loro «vantaggio morale».

Self-interest. Una visione molto diffusa nelle scienze sociali e politiche considera il self-interest il motore delle scelte e delle azioni umane. Secondo tale visione nelle transizioni istituzionali e politiche gli attori - individui e gruppi - sono spinti ad agire dall'anticipazione delle conseguenze future di opzioni preferite individualmente. $\mathrm{Ma}$ in situazioni di instabilità politica e sociale è difficile fissare criteri stabili per calcolare le utilità e per stabilire quali conseguenze meglio rispondono ai propri interessi. Il calcolo del self-interest è particolarmente complicato in situazioni sfuggenti, in continua evoluzione, dove le preferenze sono variabili e incoerenti, e dove c'è ampio spazio per la manipola- 
zione. Le preferenze sono influenzate in modo endogeno dai cambiamenti. C'è incertezza su quali debbano essere le preferenze nella nuova situazione. Di conseguenza, gli attori tendono ad adottare le preferenze di altri attori sulla base di un principio di imitazione, oppure attivano comportamenti conformi a norme già stabilite, cioè preferiscono ciò che ha una base normativa, indipendentemente da strette considerazioni di utilità (March e Olsen 1989) 9 .

$\mathrm{Nel}$ corso di un processo di trasformazione istituzionale emergono in modo ricorrente interrogativi sugli effetti possibili di diverse modalità di allocazione intertemporale delle risorse. I benefici più misurabili dell'adesione ai vecchi assetti vengono confrontati con quelli meno misurabili, spesso ignoti, della scommessa sul nuovo. Vengono valutati i tempi di attesa e la capacità di avere pazienza. Ci si domanda in che misura le scelte e le azioni presenti influenzeranno gli esiti futuri. Questi interrogativi non trovano facili risposte sulla base di un semplice calcolo dei trade-offs tra i presenti criteri di utilità e i criteri di utilità in momenti futuri relativi a uno spettro di possibili assetti istituzionali alternativi. I confronti intertemporali delle utilità sono difficili per almeno tre distinte ragioni:

- primo, perché l'incertezza è elevata ed è difficile calcolare i vantaggi relativi associati con diversi assetti istituzionali o insiemi di regole futuri, che sono ignoti e intrinsecamente instabili;

- secondo, perché l'utilità futura di un attore individuale relativa a una specifica opzione istituzionale è essa stessa una funzione di effetti di aggregazione «emergenti», vale a dire di azioni non concertate e interdipendenti che si manifestano nel corso del processo di costruzione;

- terzo, perché l'utilità futura dipende anche dal tipo di istituzioni che vengono edificate nel breve termine.

Illustriamo più diffusamente. Il flusso di benefici futuri per un attore dipende anche dalle scelte e dalle azioni degli altri attori. Se mi oriento verso un'opzione a lungo termine che richiede la collaborazione di altri, ma la collaborazione non viene offerta, è assai improbabile che l'opzione si materializzi. Di conseguenza non otterrò i benefici futuri e, inoltre, perderò i benefici

9 È necessario far notare tuttavia che nelle crisi e nelle transizioni istituzionali il comportamento guidato da regole è reso problematico dall'ambiguità, dalla molteplicità, e dall'instabilità delle regole stesse. C'è conflitto sulle regole, sui valori e sugli assetti che venivano dati per scontati in precedenza. 
di cui gli altri eventualmente godranno nel breve termine. In altri termini, potrò ottenere maggiori benefici dal mio investimento nel lungo termine solo se tutti gli altri si orientano al lungo termine. Al contrario, se perseguo il mio interesse nel breve periodo, come molti altri fanno, il risultato sarà che la profondità temporale della funzione di utilità si ridurrà, la probabilità di elevati ritorni futuri si abbasserà, e il consolidamento istituzionale nel lungo termine sarà ritardato.

Per tali motivi, nei processi di transizione gli attori mostrano cautela nell'abbandonare lo status quo, anche in presenza di esternalità negative, perché c'è incertezza sugli stati futuri e su quali utilità, individuali e aggregate, saranno associate ad essi ${ }^{10}$. Lasciare lo status quo significherebbe entrare in una fase confusa in cui si devono negoziare nuove regole - fase la cui durata non può essere stabilita ex ante, con conseguente instabilità. Gli attori sono cauti anche nel ristabilire regole specifiche, perché possono immaginare possibili regole alternative che potrebbero portare a maggiori vantaggi. Le negoziazioni implicite ed esplicite sulle regole quando le regole sono in discussione sono caratterizzate da incertezza più elevata rispetto alle negoziazioni su variazioni marginali delle regole quando le regole sono condivise.

Da una parte la convergenza di interessi multipli nella realizzazione di una struttura istituzionale nel breve termine può essere necessaria per poter edificare un' «impalcatura» o un «ancoraggio» utile alla costruzione di un framework istituzionale nel lungo periodo, ma d'altra parte essa potrebbe condurre a strutture e a sistemi di regole vulnerabili e intrinsecamente instabili, essendo prive di molte importanti proprietà necessarie alla riproduzione e al consolidamento nel lungo periodo.

In breve, in situazioni dinamiche di crisi e di ricostruzione istituzionale, nelle quali capita sovente che le scelte e gli impegni precedenti vengano smentiti dai successivi, il perseguimento del self-interest - sia nel breve che nel lungo termine - può portare a condizioni che lo rendono self-defeating. Ne può risultare

10 Questo pattern di comportamento è in qualche modo collegato a ciò ch'è stato chiamato, in modi diversi, «effetto dote» (endowment effect), status quo bias, o «avversione alla perdita» (loss aversion): individui che fronteggiano scelte ad elevato rischio percepiscono la disutilità della perdita di (o rinuncia a) un oggetto o stato che ha valore come più elevata dell'utilità associata alla sua acquisizione. L'asimmetria di valore rinforza le preferenze nella direzione dello status quo. Si veda Kahneman, Knetsch e Thaler (1991). 
un rafforzamento della stabilità o, alternativamente, un aggravamento dell'instabilità.

Identità. In termini più radicali, le difficoltà di definire una funzione di utilità chiara e stabile nel tempo corrispondono alle difficoltà di definire la propria identità futura. Come Pizzorno (1986a) fa notare, la comparazione intertemporale degli interessi di una persona o di un gruppo è possibile solo se la loro identità resta stabile. Altrimenti diventa illusorio comparare benefici attribuiti a diverse identità che possiedono valori differenti e spesso incompatibili in momenti temporali diversi. Ma per assumere un'identità intertemporalmente stabile e riconoscibile si deve assumere a fortiori un ordine istituzionale minimale. In altri termini, in assenza di istituzioni l'identità è confusa, e quando l'identità è confusa la scelta diventa problematica. Nella creazione di istituzioni il problema della scelta intertemporale basata sul self-interest si confonde col problema dell'identità.

Nei processi di crisi e di ricostruzione istituzionale le identità delle persone e dei gruppi sociali vengono scosse e allo stesso tempo nuove identità prendono forma. Gli attori sono disorientati sia sulla propria identità che sulle implicazioni dell'identità per l'azione in situazioni specifiche. Poiché l'identità di ciascun attore è interdipendente con le identità degli altri, la confusione di ciascun attore accentua la confusione degli altri. L'esplorazione delle identità possibili è complicata e rallentata dall'interazione. Più drammaticamente, assai spesso la formazione di nuove identità può avvenire solo attraverso la distruzione delle vecchie. Come conseguenza, identità relativamente «locali» (cioè con poche interazioni) e inoffensive (per quelle già consolidate) vengono ristabilite prima, mentre strutture di riconoscimento e di obbligazione più complesse emergono più lentamente, vengono ricostruite a partire da strutture più elementari, tendono a includere identità preesistenti e a ricombinare, inavvertitamente o deliberatamente, molte caratteristiche e regole precedenti.

I processi di crisi e di ricostruzione istituzionale pongono inoltre requisiti contraddittori sull'identità individuale e collettiva. Da una parte essi richiedono la capacità di rompere con il passato, trascendendo la propria identità per «convertirsi» e «rinascere» ad una nuova, emergente identità; dall'altra essi richiedono la capacità di «gettare un ponte» tra il passato (o il 
presente) e il futuro, ristabilendo così un senso di continuità. Sia la discontinuità che la continuità hanno valore e vengono cercate, ma esse si escludono mutuamente: è difficile assicurare l'una e l'altra simultaneamente. (Si noti tuttavia che a volte una continuità può essere stabilita attraverso l'opposizione e il contrasto tra il vecchio e il nuovo $)^{11}$.

$\grave{E}$ difficile creare istituzioni se non emergono a un tempo anche solide identità collettive. Ma queste a loro volta possono emergere solo se vengono riconosciute tali da un sistema sociale di riconoscimento sostenuto da e incorporato in un framework istituzionale, che è esso stesso il prodotto del processo di costruzione istituzionale. L'identità che deve essere riconosciuta e il sistema di riconoscimento dipendono l'uno dall'altro: essi emergono nel corso dello stesso processo (Pizzorno 1986b). La certezza di riconoscimento degli Io futuri, «cerchie» o gruppi stabili, e complessi sistemi di regole e obbligazioni possono darsi solo attraverso un commitment collettivo e l'emergenza endogena di una identità collettiva che si riproduca nel tempo - tutte condizioni che, come sopra abbiamo visto, sono difficili da realizzare nelle incertezze e nelle ambiguità di una crisi.

Se un attore si obbliga a un Io futuro facendo un forte investimento in valore, egli deve rompere con i suoi Io passati e presenti e «convertirsi». Se cerca sicurezza di riconoscimento nel futuro, deve sacrificare la propria sicurezza e la propria identità nel presente, vale a dire un certo grado di connettività interpersonale: l'Io attuale diventa solo uno «strumento» per il futuro. Ma se abbandona il suo Io attuale troppo presto, rischia di perdersi in una terra di nessuno, nella quale il riconoscimento sociale è problematico - un rischio che potrebbe non sentirsi di correre. E tuttavia un progresso verso una nuova identità può essere realizzato solo nella misura in cui ci si spoglia progressivamente dell'identità attuale.

L'identità - individuale o collettiva - è una sorta di costo immerso: più elevato è l'investimento (cognitivo, normativo, ideologico, emotivo, esistenziale) che gli individui o le organizzazioni fanno in una specifica identità ( o in una cerchia di riconoscimento $o$ in un sistema di regole), più forte sarà il senso di attaccamento e di lealtà a quella identità, e conseguentemente

11 Secondo Pizzorno (1995) l'identità implica la capacità di immaginare se stessi come diversi dagli altri e continui nel tempo. 
più alto sarà il prezzo che essi dovranno pagare per abbandonarla e per assumerne una differente quando sarà necessario farlo (Pizzorno 1986b). Le identità, così come le istituzioni, esibiscono resilienza e proprietà inerziali. Quando si radicano profondamente e per lungo tempo diventano difficili da cambiare. Questo è il motivo per cui rompere con il proprio Io attuale e con lo status quo è nella maggior parte dei casi un passo assai delicato: implica la capacità di rompere anche con molti degli altri Io attuali e di obbligare se stessi agli Io futuri - propri e altrui - che sono ancora sconosciuti. Viene in tal modo minacciata la percezione della continuità dell'Io nel tempo. C'è sempre il potenziale pericolo di «cessare di esistere» - una possibilità che nelle crisi e nelle transizioni politiche gli attori sociali e politici percepiscono assai chiaramente.

Se consideriamo la dinamica dell'identità, la costruzione di istituzioni implica sempre un qualche grado di incoerenza tra gli Io passati (e presenti) e gli Io futuri degli attori. Si desidera la trasformazione e l'innovazione e allo stesso tempo si vuole assicurare un qualche grado di coerenza e di accountability per le identità individuali e collettive. Così, costruire un'istituzione significa sempre formare e consolidare nuove identità, distinguibili da quelle passate, ma significa anche stabilire una qualche sorta di legittimità delle nuove identità e strutture, basate su precedenti o «esemplari» ancorati nel passato. In effetti, un modo assai comune per invocare legittimità per un nuovo progetto istituzionale è appellarsi a qualche modello legittimo già conosciuto che ha mostrato la sua validità ed efficacia in passato (Offe 1992). L'argomento della «familiarità» sembra essere assai forte per promuovere la legittimità e l'accettazione di un modello istituzionale. Il dilemma per qualsiasi framework istituzionale innovativo, come per esempio una nuova costituzione politica, è che esso deve essere «diverso» dal passato - una vera rottura rispetto al vecchio regime - e allo stesso tempo «discendente» da (o simile a) un archetipo storico rilevante. La discendenza da (e la conformità a) un modello a cui viene riconosciuto valore è l'unico modo per assicurare legittimità e stabilità in situazioni altamente incerte, specialmente quando la «prestazione» e i benefici collettivi non hanno avuto ancora il tempo di manifestarsi e dunque non possono essere invocati come fonti di legittimità per il nuovo assetto.

Il dilemma dell'identità, che ho sopra brevemente descritto, viene esperito dagli attori sociali e politici in una varietà di forme 
e viene espresso in un vasto spettro di risposte comportamentali. Nelle transizioni politiche gli attori sono per così dire presi in mezzo tra le vecchie e le nuove identità. Criteri di appropriatezza per l'azione non sono facilmente disponibili. Gli scambi, le obbligazioni, e i calcoli di utilità non possono contare sulla stabilità delle identità. Di conseguenza possiamo spesso osservare fenomeni come ad esempio oscillazioni tra identità differenti e confuse; formazione di identità locali, effimere e sfuggenti; nuove identità e affiliazioni «abborracciate» che risultano dalla ricombinazione e dalla clonazione delle preesistenti; emergenza di soluzioni «finte» e «ibride»; diaspore, eresie, e scissioni. Le risposte possono essere divise tra risposte divergenti, con un meccanismo che separa le identità o le converte l'una nell'altra (scissione, schizogenesi) e risposte convergenti, con un meccanismo che selettivamente combina identità multiple o i loro tratti componenti (combinazione, clonazione, ibridizzazione). I comportamenti erratici, oscillatori, spesso incomprensibili che gli analisti a volte hanno modo di osservare negli attori politici nel corso di una seria crisi istituzionale o politica sono dovuti meno a scelte strategiche o a tattiche deliberate e molto di più all'instabilità, alla volatilità e alla ricombinazione delle identità. Essi sono sintomi in cui si manifesta il dilemma dell'identità ${ }^{12}$.

Fiducia. Una crisi istituzionale cambia la percezione individuale e collettiva dei rischi implicati dalle scelte e di conseguenza la disposizione a concedere fiducia a persone e a istituzioni nel breve e nel lungo termine. Essa turba l'equilibrio di fiducia interpersonale e fiducia istituzionale, che in diverse combinazioni caratterizza la società in situazioni «normali» ${ }^{13}$. La debolezza

12 In questa linea di interpretazione, l'ideologia può essere interpretata come un mezzo per gettare un ponte verso stati futuri e per agganciare al lungo termine identità instabili quando nessun altro modo è possibile (proiettando nel futuro ciò che si vorrebbe essere nel presente). Creando e imponendo una rigidità di comportamento, l'ideologia riduce la necessità di apprendimento nel breve periodo, contribuendo a stabilizzare una qualche sorta di identità collettiva. Nei termini usati da Jon Elster, l'ideologia è insomma per una comunità un modo per autolegarsi, mediante un pre-commitment, ad una identità futura (Elster 1984).

13 Per questa distinzione concettuale, e per le diverse funzioni dell'uno e dell'altro tipo di fiducia, si veda Niklas Luhmann (1979). La fiducia istituzionale differisce dalla fiducia interpersonale in quanto non implica un legame personale specifico tra le persone, ma richiede la fiducia generalizzata in un insieme comune di regole in cui tutti abbiano fiducia. Come Luhmann (1979) e Gambetta (1988b) hanno sottolineato, la fiducia istituzionale richiede «fiducia nella fiducia». Sebbene ci siano importanti connessioni tra le due, prendo qui in esame solo la fiducia istituzionale. 
o l'assenza di regole istituzionali condizionano (e a loro volta sono condizionate da) l'allocazione intertemporale della fiducia. Quando un regime crolla, le regole istituzionali vigenti fino a quel momento vengono sospese o diventano inaffidabili, ma nuove regole non sono immediatamente disponibili, e devono essere istituite e applicate. Diventa allora più difficile trovare situazioni e controparti che meritino fiducia. Atti come assumere impegni credibili, onorare contratti, fare promesse diventano problematici. Lo spettro di azioni possibili per gli attori subisce una restrizione e l'orizzonte temporale per contrarre obbligazioni future tende ad accorciarsi. La fiducia nelle istituzioni diventa allora una risorsa scarsa proprio quando essa sarebbe maggiormente necessaria per impegnarsi in investimenti nel lungo periodo che comportano rischi elevati e che richiedono agli attori comportamenti esplorativi ${ }^{14}$.

Nell'esplorazione di configurazioni istituzionali alternative, dove i rischi sono elevati e gli esiti incerti e distanti nel tempo, l'accountability deve essere sostituita dalla fiducia. Il valore della fiducia come risorsa spendibile aumenta con l'aumentare del rischio e con l'allontanarsi dell'orizzonte temporale dell'azione. Infatti, quando la realizzabilità e il rendimento di assetti istituzionali alternativi non possono essere valutati con precisione, o quando non è possibile determinare gli effetti nel tempo di specifiche soluzioni istituzionali, la fiducia aumenta la tolleranza nei confronti dell'incertezza. E tuttavia la fiducia emerge con fatica in situazioni, come tipicamente sono le transizioni politiche e istituzionali, che presentano un'elevata instabilità e mortalità delle regole. Se le regole cambiano frequentemente e se non si può essere certi ch'esse dureranno abbastanza a lungo in futuro, esse non verranno percepite come una base solida per intraprendere azioni e per stabilire accordi ${ }^{15}$. La formazione di

14 Il fallimento di esperimenti istituzionali a grande scala produce al tempo stesso sfiducia e il bisogno estremo di fiducia. Secondo il sociologo polacco Piotr Sztompka, la cultura pervasiva della diffidenza è il più serio ostacolo alla ricostruzione istituzionale nelle società post-comuniste dell'Est europeo (Sztompka 1996). Esempi di processi storici di accumulazione di fiducia, che hanno avuto esiti positivi, sono invece lo sviluppo di una comunità civica democratica (Putnam 1993), o l'espansione della società commerciale internazionale (Scazzieri 1994), oppure l'introduzione di standard di misura comuni nella produzione e nel commercio (Poni 1995).

15 Nella società politica, in assenza di un sistema di regole certo, l'erosione di fiducia istituzionale viene spesso compensata da una spinta alla personalizzazione della politica e da tendenze al «leaderismo», cioè da un patto di fiducia personale tra «il popolo» e un leader carismatico. Emblematico il caso dell'America Latina. A questo proposito vedi Alberti (1996). 
aspettative comuni diventa problematica. Pochi scommetteranno sul lungo termine. Il riferimento collettivo generalizzato a un sistema di regole non viene incoraggiato quando l'orizzonte di vita del sistema tende ad accorciarsi. Una pressione inflazionistica alla proliferazione di regole ha così l'effetto di erodere la validità, la legittimità e l'efficacia delle regole stesse. Ciò favorisce le violazioni e dà adito all'ulteriore produzione di regole. Un codice instabile e attraversato da continue modifiche perde presto in affidabilità e legittimità, perché gli investimenti morali e simbolici fatti nel codice non hanno il tempo di generare un flusso di benefici e di alimentare la fiducia, estendendo nello spazio e nel tempo le possibilità e le opportunità d'azione per la collettività. In queste condizioni la creazione di capitale sociale, nella forma di istituzioni e codici generalizzati, diventa problematica.

Nell'esplorazione di nuove configurazioni istituzionali sono presenti simultaneamente fattori che inibiscono e che stimolano la fiducia. Una serie di sperimentazioni fallite può abbattere la fiducia, inducendo gli attori all'apprendimento della cautela e della diffidenza, e all'eventuale ripiegamento su posizioni e soluzioni meno rischiose. La cautela può essere una virtù in presenza di rischio, ma se la cautela e la diffidenza diventano eccessive si perdono anche opportunità d'azione. Al contrario, il successo dell'esplorazione stimola la fiducia, ma può anche indurre gli attori a una eccessiva confidenza nelle proprie capacità e all'illusione infondata di poter facilmente e rapidamente realizzare audaci progetti di riforma e di design istituzionale che non trovano poi riscontro pratico. Come si sa, l'eccessiva confidenza o la fiducia infondata abbassano il livello di attenzione e l'interesse cognitivo all'esplorazione. Vengono di conseguenza sottovalutati i rischi implicati in situazioni potenzialmente pericolose. Quando ciò avviene, crescono le probabilità di fallimento.

Il consolidamento della fiducia istituzionale ha dunque bisogno di essere sostenuto da attività di exploitation, vale a dire dallo sfruttamento e dal perfezionamento adattivo di un sistema di regole. L'exploitation è un'attività a basso rischio, e richiede quindi investimenti iniziali in fiducia relativamente meno impegnativi dell'exploration, perché l'apprendimento e il cambiamento sono più graduali e non ci sono salti drammatici a sistemi di regole radicalmente nuovi che potrebbero avere costose conseguenze impreviste. In effetti la fiducia è uno dei tanti $b y$ products che scaturiscono dallo sfruttamento prolungato di un 
sistema di regole stabile e condiviso. Come Albert Hirschman ha osservato, la fiducia aumenta con l'uso e la diffusione nella società. Ma un'eccessiva exploitation, o un'exploitation che per lungo tempo abbia prodotto esiti positivi, può avere anch'essa controindicazioni. In primo luogo un'elevata specializzazione del sistema di regole comporta un tipo di fiducia che può essere spesa solo nell'ambito di un assetto specializzato, non al di fuori. In secondo luogo, quando l'exploitation ha eccessivo successo, può condurre gli attori ad un eccesso di confidenza in assetti istituzionali o codici che col tempo potrebbero rivelarsi troppo rigidi e specifici, diventando così vulnerabili nel lungo periodo. Anche in questo caso l'eccessiva confidenza nello status quo tende ad escludere la possibilità e l'esplorazione di assetti istituzionali alternativi.

Per poter costruire istituzioni, e soprattutto per mantenerle, è necessario apprendere ad avere fiducia e a controllare selettivamente le modalità con cui essa deve essere concessa o sospesa. Apprendere ad avere fiducia significa apprendere a valutare le conseguenze positive e negative delle diverse modalità di allocazione (intertemporale e intergruppo) della fiducia ${ }^{16}$. Ciò richiede sia exploration che exploitation. Tuttavia proprio gli stessi processi che generano la fiducia o una «sana cautela» possono anche generare eccessiva confidenza o eccessiva cautela, le quali entrambe hanno conseguenze controproducenti per la costruzione di istituzioni ${ }^{17}$.

16 Occorre notare tuttavia che tali conseguenze possono riguardare non solo l'ordine economico o politico della società, ma anche il suo ordine morale. Quindi l'apprendimento della fiducia (o della diffidenza) non è un'attività puramente strumentale, «calcolativa», ma implica un orientamento e un'apertura morale (o, alternativamente, una chiusura).

17 Tra fiducia e sfiducia esiste però un'importante asimmetria. L'apprendimento della fiducia è un processo lento e faticoso, spesso non intenzionale, che produce regole, credenze, e convincimenti estremamente vulnerabili alla distruzione deliberata: una volta che la fiducia è stata tradita, è assai difficile ripristinarla. Al contrario, l'apprendimento della diffidenza è molto rapido e genera convincimenti e comportamenti difficili da sradicare. Una volta che la cultura della diffidenza è stata appresa, essa cerca continuamente conferme. Il sospetto tende ad essere più contagioso della fiducia. Distruggere fiducia è dunque molto più facile e rapido che ricostruirla (Pagden 1988; Sztompka 1996). 


\section{Meccanismi costruttivi}

La trattazione sviluppata fino a questo punto può aver trasmesso l'idea che la costruzione di istituzioni sia un'impresa assai ardua. Come ho cercato di mostrare, un certo numero di fattori - incertezza sul futuro, avversione al rischio, sunk costs, disparità tra breve e lungo periodo, competenze e risorse specializzate, problemi distributivi nel tempo e tra i gruppi, identità ambigue, mancanza di fiducia, spesso in combinazione o in interazione tra loro - generano una complessa dinamica che rende difficile la sostituzione dei vecchi assetti istituzionali con $\mathrm{i}$ nuovi. Individui e società sembrano preferire strategie conservative e a basso rischio, rivelando un forte bias per l'exploitation. $\mathrm{Ma}$ neppure questo li salva da conseguenze non desiderate e dalla possibilità dell'autodistruzione. Dunque i processi di costruzione delle istituzioni non danno solo luogo a esiti possibilmente instabili e fragili, ma anche a esiti perversi.

Nessuna società può d'altra parte sopportare i costi necessari per smantellare il suo apparato istituzionale tutto in una volta. Raramente la sostituzione completa di assetti istituzionali, per quanto obsoleti e cadenti, è una strategia fattibile o sicura. Una deviazione improvvisa e radicale dalle pratiche e dalle strutture abituali risulta troppo rischiosa, e gli attori politici e le loro constituencies non la accetterebbero. La sfida è troppo grande, sia per gli innovatori, rivoluzionari o riformisti, che per i conservatori, i guardiani dello status quo. Le innovazioni sociali e politiche troppo radicali comportano un elevato potenziale di conflitto e di divisione, al punto che possono diventare incontrollabili e distruttive nel lungo periodo. Tuttavia, nonostante queste difficoltà la costruzione di istituzioni «accade» attraverso una notevole varietà di meccanismi sociali (Abell 1995), ed è un processo continuo ed estremamente diffuso ${ }^{18}$. L'ultima parte del lavoro si concentrerà allora sulle seguenti domande: come è possibile la costruzione di istituzioni? Come è possibile aggirare i dilemmi e i cicli autodistruttivi nell'allocazione intertemporale delle risorse e alimentare un processo costruttivo? C'è una via d'uscita al dilemma tra exploration e exploitation?

18 Le istituzioni possono emergere come forme cristallizzate di reticoli sociali o patterns di attività preesistenti, da movimenti sociali, o emergere sotto la spinta dell'ambiente esterno, o risultare da una organizzazione preesistente, o anche dall'azione di un imprenditore istituzionale dotato di carisma. 
Mi limiterò a esaminare tre importanti modalità o meccanismi che operano nei processi costruttivi, specificatamente i punti focali, i rendimenti crescenti, e il bricolage istituzionale. I punti focali riguardano l'emergenza del coordinamento, i rendimenti crescenti si riferiscono ai processi di rinforzo e di diffusione di regole e di patterns di comportamento, il bricolage connota la costruzione e l'evoluzione di macrostrutture a partire da strutture più modeste. Questi meccanismi, tra gli altri, costituiscono risposte efficaci alle dinamiche autodistruttive e modi per risolvere o superare alcuni dei dilemmi dell'azione finora discussi. Per far fronte alle difficoltà e alle incoerenze del cambiamento sociale e politico, gli attori impegnati o coinvolti nella costruzione di istituzioni tendono ad attivare, più o meno consapevolmente, meccanismi e strategie che meglio permettono loro di ridurre l'incertezza e il rischio, di economizzare sui costi immersi, di attenuare i conflitti intertemporali e intergruppo, di tagliare i costi di transizione e di apprendimento, di stabilizzare identità, fiducia e significati. Cì̀ che qui sostengo è che i punti focali, i rendimenti crescenti, e il bricolage sono meccanismi che adeguatamente rispondono ai suddetti requisiti e possono essere osservati in un vasto spettro di casi empirici di costruzione e mutamento delle istituzioni ${ }^{19}$.

Punti focali. Come Schelling lo ha definito, un «punto focale» è una caratteristica prominente, cospicua, di una situazione ambigua di scelta che, proprio per la sua unicità e visibilità, diventa una chiave, o piuttosto la chiave per risolvere un difficile problema di coordinamento:

il rinvenimento di una chiave - una qualsivoglia chiave che sia mutuamente riconosciuta come la chiave diventa la chiave - può dipendere dall'immaginazione più che dalla logica; può dipendere dall'analogia, dal precedente, da un assetto accidentale, dalla simmetria, dalle proprietà estetiche o geometriche di una configurazione, da un ragionamento casuale, e anche dalle identità di

19 Non intendo sostenere che questi sono gli unici meccanismi o strategie per rispondere ai problemi e ai dilemmi della costruzione di istituzioni, come sono stati illustrati in questo lavoro, e tanto meno che sono gli unici modi con cui le istituzioni vengono formate. Ce ne possono essere altri, dalla progettazione intenzionale ai processi evolutivi endogeni, a una combinazione di ingegneria istituzionale e evoluzione sociale. La mia tesi è che $\mathrm{i}$ punti focali, i rendimenti crescenti, e il bricolage ci aiutano a interpretare parecchi fenomeni che hanno luogo nei processi di costituzione delle istituzioni - fenomeni che, io credo, non ricevono la giusta attenzione dagli scienziati sociali e politici né dai legislatori e progettisti costituzionali. 
quelli che si confrontano e dalla conoscenza ch'essi possiedono l'uno dell'altro (Schelling 1980, 57).

In situazioni strategiche, dove molteplici attori con interessi conflittuali sono coinvolti in complessi processi decisionali, considerazioni di «ovvietà» o «visibilità» di una soluzione possono prendere il sopravvento su considerazioni di interesse personale (Kreps 1990; Garrett e Weingast 1993). L'unicità di un punto focale induce «naturalmente» un certo grado di convergenza e coordinamento. Lo fa riducendo l'ambiguità sulle soluzioni possibili e creando un punto di riferimento per gli attori. Un punto focale incapsula pochi fondamentali requisiti informativi che tutti conoscono e possono utilizzare per interpretare una situazione e orientare l'azione. Si convergerà ad una specifica soluzione perché ci si aspetta che altri vi convergeranno a loro volta, indipendentemente da calcoli razionali sugli interessi presenti e da aspettative sulle utilità attese future. In situazioni di collasso istituzionale, dove a causa del disordine ambientale la comunicazione è difficile e la convergenza su un assetto istituzionale condiviso è problematica, i punti focali possono offrire soluzioni extra-razionali, ma «ovvie», al problema della coordinazione sociale ${ }^{20}$.

I punti focali emergono in genere in modo accidentale, spesso non intenzionalmente, ma a volte, come Garrett e Weingast (1993) hanno suggerito, possono risultare da una costruzione o da una ricerca collettiva. Nell'uno o nell'altro caso è estremamente difficile e può addirittura essere controproducente dire ex ante ciò che costituisce un punto focale, e se funzionerà come tale. Nella costruzione di istituzioni i punti focali possono assumere diverse forme, a seconda della cultura, delle pratiche passate, delle istituzioni esistenti, o delle routine organizzative correnti. Possono essere forniti da leader politici o da padri fondatori, da credenze e principi condivisi, da movimenti sociali, emozioni collettive, assetti ereditati, expertise tecnica, dal commitment eccezionale di un gruppo, da un enclave di profon$\mathrm{da}$ fiducia interpersonale fondata su un'identità collettiva di tipo etnico, di genere, di ruolo o di altro tipo. Un punto focale può anche consistere in un qualche ordinamento costituzionale

20 «Extra-razionale» non significa qui «irrazionale» né «non-razionale»; significa che le soluzioni non possono essere spiegate o predette sulla base della teoria della scelta razionale, cioè attribuendo motivazioni di interesse personale ad attori razionali. 
o politico che funge da modello, da una struttura di credenze che acquista valore normativo e una legittimità riconosciuta, o altrimenti da uno schema o unità narrativa archetipica che diventa una fonte generativa per la riproduzione di senso e di struttura, generando così credenze e patterns d'azione istituzionalizzati ${ }^{21}$. Una volta emersi, i punti focali diventano meccanismi «pivotali» per coordinare aspettative e comportamenti - catalizzatori che trasformano la politica.

La letteratura ha finora sottolineato prevalentemente il ruolo dei punti focali nel risolvere i problemi degli equilibri multipli e della contrattazione incompleta nell'ambito della teoria dei giochi (Garrett e Weingast 1993; Kreps 1990). Quando ci sono molteplici vie alla cooperazione ma nessuna forma di convergenza è accettabile per gli attori, l'emergenza di un punto focale può costituire la soluzione «ovvia» ${ }^{22}$. Piuttosto che il problema «orizzontale» della cooperazione vorrei qui mettere in luce il ruolo dei punti focali nell'attenuazione dei conflitti che nascono nell'allocazione intertemporale delle risorse. In che modo essi permettono di uscire dai dilemmi e dai cicli di fallimento che ho sopra schematicamente illustrato? In che modo i punti focali interrompono la dinamica autodistruttiva nella costruzione di istituzioni?

La mia tesi è che i punti focali possono essere «germi» per la strutturazione di assetti istituzionali nel corso del tempo. Essi non sono necessariamente soluzioni stabili che vengono «trovate», ma piuttosto «segnali» che puntano in una direzione - dove guardare in futuro. Se nel bel mezzo di una situazione di destrutturazione o di crisi emerge o viene costruito un punto focale, intorno ad esso prende forma un campo ordinato che dà agli attori un senso di orientamento e di commitment. Che lo vogliano o no, gli attori devono porsi in relazione con esso, devono in

21 Come esempio si consideri la famosa spiegazione di Arnold Toynbee della «storia di Gesù» e delle sue capacità riproduttive e diffusive, simili a quelle di un virus, in una varietà di differenti culture (Toynbee 1934-1959).

22 Garrett e Weingast hanno applicato la nozione di focal point allo studio del processo decisionale multi-attore che ha portato alla creazione del mercato interno dell'Unione Europea alla metà degli anni ottanta. Secondo gli attori, erano disponibili diverse soluzioni istituzionali per sostenere il mercato, e i partners non avevano nessuna preferenza dominante o specifica per qualcuna di esse. La soluzione focale fu data dalla decisione del 1979 della Corte Europea di Giustizia, che asseriva che il riconoscimento reciproco tra i paesi-membri doveva essere parte integrante della legge comunitaria. Questo «precedente» fu infine scelto dai partners Europei come il principio-guida per ordinare il mercato interno. 
un certo senso «passarci attraverso», e sospendere il cammino alla cieca. Un punto focale ha proprietà magnetiche. Mette in movimento forze che producono strutture stabilizzanti: i comportamenti vengono alterati e un ciclo convergente di attese e azioni viene attivato. L'evento-chiave implica un'aspettativa che genera un'azione che causa la materializzazione di un risultato che conferma quell'aspettativa. In questo senso, i processi di costruzione di istituzioni, quando hanno successo, sono sempre in una certa misura profezie che si autoadempiono. Creando convergenza nel breve periodo, un punto focale stabilizza una struttura minimale che, via via che guadagna momento, può dare un senso di organizzazione e di direzione nel lungo periodo. Per poter operare come un evento generatore di forma, un punto focale deve essere associato con un meccanismo positivo di rinforzo, che interrompe i cicli di instabilità. Nell'espressione di Karl Weick, «a small structure goes a long way» (1993).

Nella prospettiva assunta in questo saggio i punti focali sono riguardati come «strutture percepibili minimali» (Weick 1995) che in situazioni caotiche possono costituire un' «àncora» per la definizione di identità, significati e riconoscimenti reciproci, una base su cui possono essere costruite fiducia e competenze, e un nucleo intorno a cui possono essere attivate risorse sempre crescenti. Un punto focale è insomma un piccolo inizio, a partire dal quale può essere attivato un ambiente dotato di senso (Weick 1993). È una struttura minimale che è potenzialmente perfezionabile, mentre al tempo stesso offre un sentiero o un campo ordinato per l'esplorazione, che altrimenti procederebbe per così dire «al buio». Fornendo un canale per l'esplorazione, un punto focale riduce $\mathrm{i}$ rischi percepiti e $\mathrm{i}$ costi ad essa associati, ed eventualmente aiuta gli attori a superare mere considerazioni di utilità nell'allocazione delle risorse, come per esempio il peso dei sunk costs. Inoltre, istituendo un pattern di aspettative ordinate nel tempo, che possono estendersi nel futuro, i punti focali permettono il coordinamento dei molteplici orizzonti temporali degli attori. Mentre forniscono un terreno su cui poggiare nel breve periodo, essi accrescono la salienza e la visibilità, dunque la raggiungibilità del lungo periodo.

Tuttavia i punti focali, come «meccanismi d'accensione» di un processo di costruzione di istituzioni, hanno i loro limiti. In primo luogo, come tutti i germi, sono vulnerabili: se non sono alimentati da un rinforzo positivo da parte dell'ambiente, potrebbero non essere in grado di produrre forma. In secondo 
luogo, possono essere volatili ed effimeri: emergono, durano per breve tempo, e poi svaniscono. In terzo luogo, possono cambiare nel corso della transizione. Infine, può darsi che ce ne sia più d'uno e che siano in competizione l'uno con l'altro. I punti focali hanno effetti di strutturazione quando sono unici. $\mathrm{Ma}$ quando sono numerosi e in competizione tra loro, e intorno ad essi si cristallizzano forti rigidità ideologiche e politiche, allora possono esserci diversi tipi di soluzioni altrettanto «ovvie», sulle quali gli attori non trovano un accordo. In simili situazioni la coordinazione e l'ordinamento intertemporale non sono assicurati, ed è probabile che il processo di costruzione di istituzioni sia altamente conflittuale. Come Garrett e Weingast hanno suggerito, la rilevanza e l'impatto dei punti focali dipende in ultima analisi dalla struttura delle interazioni tra gli attori: $i$ punti focali sembrano avere più peso sugli esiti del processo quando le asimmetrie distributive e il differenziale di potere tra gli attori sono minori (Garrett e Weingast 1993, 186).

Rendimenti crescenti. In una semplice (e forse semplicistica) caratterizzazione le istituzioni possono essere riguardate come il risultato di un processo di apprendimento basato su scelte sequenziali e su un feedback positivo di rinforzo e amplificazione. Partendo da eventi fortuiti o da strutture minimali che valgono come punti focali, il feedback positivo permette la formazione e il consolidamento di strutture più grandi e più stabili, che danno rendimenti crescenti (Arthur 1988; David 1985 e 1992). Esso è un meccanismo attraverso il quale l'esperienza passata o «storia» viene accumulata in strutture, regole, e routine - formali e informali - fino a costituire un modello di riferimento per il funzionamento, il significato, e l'identità (Etheredge 1976; March e Olsen 1984; Levitt e March 1988; North 1990) ${ }^{23}$. Nel corso del processo, le strategie, le competenze, le aspettative, e

23 Tale meccanismo tuttavia non è necessariamente efficiente né conduce a soluzioni ottimali: le istituzioni, passate e presenti, non costituiscono il migliore dei mondi possibili (Unger 1987). Ciò accade solo nelle utopie. L'apprendimento è spesso limitato (Argyris e Schon 1996) o miope (Levinthal e March 1993). Le inferenze e la memorizzazione dell'esperienza passata possono essere distorte o inadeguate, e produrre esiti e comportamenti subottimali. Gli adattamenti prodotti dai processi di apprendimento possono rivelarsi inappropriati o malaccorti, troppo rapidi o troppo lenti, generando così conseguenze controproducenti. Nell'espressione di Levinthal e March (1993, 97): «l'apprendimento ha le sue proprie trappole». Inoltre i processi di apprendimento possono essere improvvisamente interrotti da eventi casuali, che annullano bruscamente l'esperienza accumulata e costringono a ripartire quasi da zero. 
le relazioni tra di esse vengono endogenamente modificate (March e Olsen 1984, 745-746). Reiterando le scelte gli attori apprendono a conoscere le loro preferenze e i loro criteri di utilità, ed eventualmente a convergere su esiti collettivamente condivisi. In tal modo le istituzioni, mentre vengono costruite, aiutano gli attori a scoprire, a perseguire, e a far convergere $\mathrm{i}$ loro interessi e le loro identità.

Il processo è basato sulla mutua interazione. Come gli attori individuali apprendono dalle istituzioni, così le istituzioni apprendono dagli individui, e i due processi sono simultanei. Nell'interazione è cruciale che sia mantenuta una combinazione equilibrata tra exploration e exploitation, tra diversità e affidabilità dell'esperienza (March 1991). Le istituzioni devono essere capaci di apprendere dalla varietà di comportamenti degli attori $\mathrm{e}$ incorporare il più possibile tale varietà nei loro codici e nelle loro routine, perché se esse diventano troppo selettive rischiano di trascurare esperienze potenzialmente utili. Idee e comportamenti devianti, potenzialmente innovativi, vengono allora prematuramente soffocati. Tuttavia le istituzioni non possono neppure codificare troppa diversità, perché in tal caso diventerebbero instabili e debolmente direttive, perdendo la loro funzione di canalizzazione dei comportamenti. Da parte loro gli attori devono apprendere dalle regole e dai codici forniti dalle istituzioni a produrre comportamenti appropriati in modo tale che un certo grado di coordinazione sociale e di affidabilità sia assicurato. Ma allo stesso tempo la convergenza e l'adattamento dei comportamenti degli attori ad un codice istituzionale non devono essere troppo rapidi o troppo reattivi, perché il codice rischierebbe di impoverirsi e di irrigidirsi in breve tempo. In tal caso le istituzioni non vengono alimentate dalla varietà, e la loro capacità di apprendimento si indebolisce.

Per rendere più chiara la mia argomentazione, illustrerò un processo di autorinforzo e di propagazione basato sul principio dei rendimenti crescenti, mostrando brevemente le implicazioni per la competenza, il self-interest, l'identità, e la fiducia. Si consideri l'esempio: "chiamare il mio vicino o chiamare la polizia quando bo un ladro in casa». Queste sono due soluzioni competitive al «problema del ladro in casa», che possono essere scelte in alternativa ${ }^{24}$. Supponiamo che inizialmente ci sia indifferenza

24 L'esempio ha solo una funzione pedagogica, non è un'applicazione empirica. Ma la linea di argomentazione qui seguita può essere applicata a tutti i casi in cui ci 
per l'una o l'altra soluzione e che un piccolo gruppo di individui distribuisca le sue scelte in quote uguali tra le due alternative. Se però nel corso del tempo un sempre maggior numero di individui continua a chiamare la polizia, essi impareranno a conoscere sempre di più la sua efficacia (o inefficacia), e tenderanno a utilizzarla sempre di più come una risorsa o soluzione pubblica al problema del ladro in alternativa alla soluzione privata che conta sul vicino di casa (o viceversa, in caso di inefficacia, tenderanno ad abbandonarla). Di conseguenza, le scelte ripetute degli attori trasformano il corpo di polizia in un'agenzia istituzionalizzata permanente che offre un servizio pubblico ${ }^{25}$. D'altra parte, se la polizia diventa un punto di riferimento per un numero sempre maggiore di individui, la sua organizzazione e le sue prestazioni tenderanno ad essere sostenute e rinforzate sempre di più, e la sua affidabilità tenderà ad aumentare (almeno fino ad una certa soglia, oltre la quale la sua prestazione può degradare rapidamente a causa di un sovraccarico di domande). Se dunque la polizia viene chiamata frequentemente e ripetutamente da un numero sempre maggiore di individui, essa può apprendere dalle loro richieste e dai loro bisogni, e migliorare il suo rendimento organizzandosi sempre meglio per rispondere alla varietà dei comportamenti. In quanto istituzione pubblica essa diventerà presumibilmente più articolata e svilupperà competenze distintive. Offrirà eventualmente servizi sempre più differenziati, che inviteranno a un'ulteriore utilizzazione da parte degli utenti. Al contrario, se un numero sempre maggiore di utenti rinuncia alla polizia come servizio pubblico e si affida piuttosto all'aiuto del vicino o ad altre soluzioni private, la polizia tenderà ad abbassare il proprio rendimento e a perdere legittimità, fino al punto di perdere di significato o addirittura di

sono due soluzioni «istituzionali» in competizione a un problema di interesse personale, per esempio: «affidarsi all'aiuto dei vicini oppure al servizio professionale dei vigili del fuoco quando brucia la casa», o «ricorrere alle autorità pubbliche oppure alla mafia per ottenere protezione privata» (si veda, per quest'ultimo caso, Gambetta 1992). Ho scelto l'esempio della polizia soprattutto per la sua generalizzabilità: tutte le società hanno il problema dell'ordine pubblico.

25 È implicita in questo esempio una caratterizzazione della polizia come servizio pubblico per assistere i cittadini e non come apparato di controllo, sorveglianza e repressione. Sarebbe del resto istruttivo esplorare le implicazioni di una tale differenza dal punto di vista dell'apprendimento: apprendere come rispondere più efficacemente alle richieste dei cittadini versus apprendere come esercitare un controllo più efficace su di essi, e apprendere a «saltare» dalla seconda alla prima caratterizzazione. In ciascuna delle due caratterizzazioni anche i cittadini apprendono comportamenti diversi nei confronti della polizia. 
esistere come istituzione sociale (o, nel caso sopravvivesse, diventerebbe un onere sociale piuttosto che un bene pubblico).

Continuare a chiamare la polizia quando c'è un problema è anche un modo per conferire ad essa valore e significato. Apprendendo a convergere sulla stessa soluzione istituzionale si apprende anche a convergere sugli stessi valori e significati. $\mathrm{Pa}$ rallelamente viene rinforzata la fiducia nella polizia come soluzione istituzionale efficace e legittima ai problemi di protezione privata e di ordine pubblico. Allo stesso tempo, apprendendo come utilizzare la polizia in quanto istituzione, gli utenti costruiscono la propria identità collettiva o qualche importante elemento di essa, diventando una comunità di cittadini che hanno titolo a chiamare la polizia in caso di difficoltà. Il punto qui non è tanto se un individuo otterrà o no dei benefici dall'interazione con la polizia (piuttosto che con il vicino di casa), ma piuttosto se la polizia viene riconosciuta come un attore legittimato a garantire l'ordine e la sicurezza pubblica e, di converso, se una persona viene riconosciuta o meno dalla polizia come avente diritto al servizio in quanto membro di una collettività. Sia per la polizia che per i cittadini rispondere alle mutue richieste è un modo per confermare o meno le rispettive identità e per verificare fino a che punto le azioni dell'uno e dell'altro sono coerenti con $\mathrm{i}$ valori e con l'identità di una comunità che si estende oltre i rapporti di clan o di vicinato.

Man mano che la polizia espande la sua capacità d'intervento e consolida la sua identità come agenzia istituzionale, si forma anche, tra l'istituzione e i cittadini, un sistema di riconoscimenti reciproci e di obbligazioni normative, simultaneamente a un sistema di transazioni orientate all'interesse, e i due sistemi possono rinforzarsi o indebolirsi a vicenda. Nel corso del processo due tipi di connessioni - interpersonali e intertemporali vengono facilitate ed estese. $\mathrm{Da}$ un lato un corpo istituzionale permanente, che trascende la provvisorietà delle identità individuali, rinforza il mutuo riconoscimento sostenuto da legami personali «orizzontali»; dall'altro, aiutando gli individui a proiettare nel futuro i loro interessi personali e le loro identità, esso assicura un riconoscimento intertemporale «verticale» tra gli Io presenti e gli Io futuri. In tal modo, frames cognitivi e normativi condivisi possono essere sviluppati, coordinati e riprodotti nel tempo 26 .

26 L'estensione e il rinforzo di entrambi i tipi di connettività, orizzontale e vertica- 
L'esempio mostra che la costruzione di un'istituzione, o la creazione di un qualsiasi codice o sistema di regole, può essere riguardata come un processo di mutuo apprendimento e di rinforzo reciproco tra individui e istituzione: nella misura in cui gli individui imparano ad affidarsi ad un attore organizzato come la polizia, quest'ultima si consoliderà come agenzia istituzionalizzata e apprenderà a rispondere alle richieste dei cittadini. Il processo parte da una molteplicità di transazioni debolmente connesse, alcune delle quali possono consolidarsi in una struttura di coordinamento più vasta. Una volta che un numero crescente di individui è «catturato» (locked-in) entro uno specifico assetto o relazione istituzionale, si manifestano rendimenti crescenti e si stabilizzano modelli di comportamento path-dependent. Emerge una struttura, viene istituito un sistema di regole, viene accumulata esperienza, vengono sviluppate competenze specifiche, e interessi personali, valori e identità vengono scoperti e ridefiniti nel corso del processo ${ }^{27}$. Al contrario, se la fiducia nella polizia declina, il suo rendimento istituzionale subirà un calo, e il valore dell'aiuto privato aumenterà col calo della prestazione dell'agenzia pubblica. Per dirla più semplicemente,

le, costituiscono meccanismi di istituzionalizzazione fondamentali. Quando essi non funzionano bene, l'istituzionalizzazione diventa problematica. Quando la connettività interpersonale non viene riprodotta, la società si frantuma in unità segmentate locali con interessi particolaristici. Quando non viene riprodotta la connettività intertemporale, individui, gruppi e le società nel loro complesso non riescono a proiettare l'immagine di se stessi nel futuro e non possono riprodursi nel tempo. Come conseguenza, le identità, le credenze, i valori e soprattutto le forme istituzionali non riescono per così dire a «viaggiare nel tempo».

27 Vorrei qui precisare che il meccanismo con cui gli individui apprendono a contare sull'assistenza della polizia piuttosto che su quella dei parenti o dei vicini di casa non è necessariamente basato sul calcolo e sulla scelta razionale, cioè su un criterio di utilità personale. Quando le utilità associate a scelte alternative sono ancora poco chiare e non quantificabili con precisione, quando insomma l'interesse personale deve ancora essere definito, una modalità molto comune di apprendimento è l'imitazione: si chiama la polizia semplicemente perché il vicino ha chiamato la polizia, e la cosa ha funzionato. I comportamenti che imitano o che copiano si propagano per diffusione, con un numero sempre maggiore di persone che fanno le stesse cose o adottano i medesimi tratti di comportamento. Alternativamente, si può chiamare la polizia in base a una credenza profonda nel valore di tale istituzione e nell'appropriatezza o «correttezza» di tale atto, indipendentemente da considerazioni di interesse personale o di efficienza (March e Olsen 1988). Mentre il comportamento imitativo sembra essere centrale per l'emergenza e l'espansione di una istituzione e per la scoperta dell'interesse personale e del valore, al contrario il comportamento basato su regole e orientato al valore dà conto più efficacemente di situazioni in cui istituzioni già esistenti vengono gradualmente rinforzate e riprodotte in quanto sono percepite o credute espressioni e portatrici di particolari valori. 
se un sempre maggior numero di persone si rende conto che lo sceriffo è inaffidabile e inefficace, il ruolo dello sceriffo si svuoterà gradualmente, e le persone impareranno a contare su «sceriffi privati» o su simili soluzioni per perseguire il proprio selfinterest e per ottenere sicurezza personale, fino al punto in cui ognuno diventa lo sceriffo di se stesso. Quando ciò accade, la comunità regredisce ad uno stato semi-selvaggio, devastata dalla diffidenza e dalla belligeranza. Dunque lo stesso meccanismo di rinforzo può operare sia nella ricostruzione di un nuovo assetto istituzionale a partire da rotti frammenti e identità confuse, sia nella distruzione e nel dissolvimento di un assetto originariamente compatto e dotato di legittimità.

Bricolage istituzionale. Raramente le istituzioni vengono create a partire da una tabula rasa. Molto più spesso esse sono il risultato della ricombinazione e del rimpasto di componenti già esistenti o di altri «materiali istituzionali» già disponibili che, pur avendo esaurito la loro funzione primaria, possono essere riutilizzati per nuovi scopi. I processi di costruzione delle istituzioni sono caratterizzati $\mathrm{da}$ attività di bricolage. Secondo Levi-Strauss, che per primo ha introdotto tale nozione nelle scienze sociali, il bricolage comporta due aspetti rilevanti (LeviStrauss 1962):

a) l'uso di materiali di seconda mano per costruire un artefatto o una struttura quando niente di più appropriato è disponibile;

b) l'utilizzo di vecchi componenti e strutture per svolgere nuove funzioni, dedicandoli a impieghi diversi da quelli per $\mathrm{i}$ quali essi erano stati originariamente progettati.

Il bricolage è un principio di progettazione e di composizione opposto ai classici principi costruttivi in voga nell'ingegneria e nell'architettura. Mentre per l'ingegneria l'ordine è il prodotto di un piano definito intenzionalmente ex ante, nel bricolage l'ordine emerge ex post come codificazione o effetto estemporaneo di cambiamenti non programmati. Intenzioni, piani, azioni e risultati sono debolmente connessi (loosely coupled). I materiali e gli strumenti del bricoleur non hanno una connessione stretta con nessun progetto particolare, ma possono essere facilmente trasposti e riorientati da un progetto preesistente ad un nuovo progetto (Weick 1993).

Se comparato al processo legislativo formale o all'ingegneria costituzionale (Sartori 1994), il bricolage istituzionale viene 
spesso riguardato come una strategia «minore». Tuttavia esso può generare conseguenze a grande scala e a lungo termine, cioè strutture che durano e funzionano. In verità, il bricolage può essere l'unico modo per creare e innovare in situazioni caratterizzate da elevata incertezza, avversione al rischio, mancanza di fiducia, conflitto politico, restrizione di risorse, e alti costi immersi. Il bricolage è insomma un modo per affrontare la complessità.

Sebbene l'importanza del bricolage nella costruzione di istituzioni non sia stata ancora esaminata sistematicamente, nella letteratura possiamo trovare alcune interessanti illustrazioni, che esaminerò brevemente ${ }^{28}$. Secondo Skowronek (1982), l'innovazione burocratica nel sistema amministrativo statunitense nel periodo 1877-1920 è avvenuta attraverso l'accostamento (patching-up) di nuovi elementi istituzionali al framework preesistente in risposta a problemi e contingenze che sorgevano via via $^{29}$. I recenti studi di Genschel sull'evoluzione del regime internazionale degli standard nel campo delle telecomunicazioni e del sistema sanitario tedesco nell'ultimo secolo illustrano come cambiamenti fondamentali nelle condizioni ambientali non abbiano provocato discontinuità istituzionali o riorientamenti radicali delle policies, ma siano stati affrontati mediante l'estensione, la trasposizione o l'integrazione di strutture e procedure istituzionali già consolidate (Genschel 1997). Guarnieri, dal canto suo, mostra come nella riorganizzazione del sistema giudiziario del Regno d'Italia dopo l'unificazione le autorità piemontesi, per applicare misure di controllo normativo, amministrativo e sociale nel Regno appena annesso delle Due Sicilie, sfruttarono astutamente le competenze già esistenti con una intelligente dislocazione, avvicendamento e riutilizzazione del personale giudiziario, togato e non, nelle diverse regioni del paese (Guarnieri 1997). Infine, come dimostrano i recenti studi di Stark sul capitalismo dell'Est europeo, il cambiamento istituzio-

28 Il bricolage può assumere la forma del «patchworking» (Skowronek 1982), dell'imitazione o della copia di assetti costituzionali come modelli esemplari per le riforme istituzionali e politiche (Lijphart 1984; Offe 1992), della trasposizione di funzioni e strutture nel campo del policymaking (Genschel 1997), o della ricombinazione e riutilizzo di componenti istituzionali e organizzativi già disponibili (Guarnieri 1997; Stark 1996).

29 Questa modalità di risposta dell'amministrazione americana è confermata da un recente studio di Baldi sulla trasformazione della U.S. Nuclear Regulatory Commission successiva all'incidente nucleare di Three Mile Island del 1979 (Baldi 1996). 
nale e organizzativo nell'Europa dell'Est è avvenuto non attraverso sostituzione radicale ma piuttosto mediante ricombinazione di routine e pratiche persistenti, o con l'emergenza di strutture parallele (Stark 1994 e 1996). Pratiche e patterns istituzionali preesistenti non sono stati cancellati bensì trasformati in assets e risorse per edificare nuovi assetti istituzionali, sia nell'economia che nella politica. Di conseguenza, il collasso delle strutture formali dei regimi socialisti non ha generato vuoto istituzionale o totale disordine, come spesso si assume. Al contrario, ha generato una situazione nella quale sono compresenti diversi sistemi di regole, spesso incompatibili, che vengono invocati per ottenere legittimazione e per giustificare l'azione.

Tutti gli studi citati si discostano dalla tradizionale iconografia dell'ordine e del cambiamento condivisa, come Orren e Skowronek (1991) hanno sottolineato, da molti analisti delle istituzioni. Essi suggeriscono l'idea fondamentale che le transizioni e le innovazioni istituzionali raramente consistono in «sostituzioni» o in «passaggi di stato», ma piuttosto in ricombinazioni e rimescolamenti di patterns. La costruzione di istituzioni allora avviene spesso non sulle rovine, ma con le rovine del vecchio regime, nel momento in cui le risorse disponibili vengono riorientate alla risoluzione di dilemmi pratici che si presentano nel corso della trasformazione (Stark 1996, 2). L'ordine istituzionale è costituito da «strati archeologici» di molteplici e diversi principi o logiche d'azione (Olsen 1992). Le istituzioni sono strutture diacroniche. Ciò che chiamiamo «ordine istituzionale» è un'intersezione di componenti istituzionali che il più delle volte non hanno una comune origine storica. L'ordine e la coerenza intertemporale tra più elementi, apparentemente incoerenti, emergono quando questi si adattano funzionalmente l'uno all'altro semplicemente per il fatto che hanno vissuto e funzionato l'uno vicino all'altro per un periodo di tempo abbastanza lungo. Gli stessi studi suggeriscono anche che la «robustezza» delle istituzioni può dipendere «dalla varietà e dal fermento piuttosto che dalla coerenza dei principi e delle procedure», e che il funzionamento efficace può dipendere dalla misura in cui le istituzioni sono capaci di sfruttare il disordine piuttosto che di assicurare l'ordine (Orren e Skowronek 1991, 329). Le istituzioni dunque non sono dispositivi sistemici per garantire ordine, o per lo meno non lo sono sempre. Piuttosto esse creano routinariamente «disordine organizzato» (patterned disorder) (Orren e Skowronek 1991, 320). 
I processi sopra illustrati sono piuttosto comuni e poco visibili, e ad alcuni osservatori potrebbero non sembrare affatto processi di cambiamento. Ma la loro scarsa visibilità può essere ingannevole. L'effetto cumulativo di piccoli cambiamenti periferici è spesso notevole, ed è proprio l'«innocenza» di ogni minimo cambiamento che rende possibile l'accumulazione di effetti su grande scala (March 1981; March e Olsen 1989). Io sostengo che ci sono ragioni non banali che spiegano perché nei casi su riportati la costruzione di istituzioni può assumere la forma apparentemente modesta del bricolage.

Se lo consideriamo nella prospettiva dell'efficienza, il bricolage permette di economizzare sui costi immersi e di proteggere gli investimenti iniziali. Dato che è troppo costoso o praticamente impossibile gettar via il capitale costituito dalle istituzioni esistenti e devalorizzare i costi immersi prima che i nuovi assetti (e investimenti) istituzionali abbiano avuto il tempo di produrre benefici, una strategia conveniente consiste nel convertire i vecchi componenti e strutture a nuove funzioni e utilizzazioni. Poiché gli esiti del bricolage sono strutture poco integrate che possono essere comunque riadattate localmente o ricombinate per far fronte a nuove contingenze, un vantaggio ulteriore del bricolage è che esso riduce la quantità di costi d'impianto incorporati nelle nuove strutture. In altri termini, il bricolage favorisce la plasticità strutturale delle istituzioni. Al contrario, nuovi frameworks istituzionali che risultano da cospicui investimenti in strutture o apparati simbolici tendono ad auto-confermarsi e a rinforzare il convincimento nella loro propria necessità e legittimità anche quando essi si rivelano chiaramente inefficienti, controproducenti e non più sostenibili (Unger 1987).

Allo stesso modo, se consideriamo i problemi della legittimità e dell'identità, il bricolage offre agli attori sociali e politici l'opportunità di restare attivi quando c'è una provvisoria perdita di significato o quando è difficile definire un'identità politica e orientarsi in una situazione di transizione. Il senso di ciò che accade nasce spesso «a cose fatte», come un by-product di ciò ch'è stato fatto, ed è sempre locale e provvisorio. Inoltre, è molto più facile per una piccola struttura ottenere legittimazione e assicurarsi una legittimità. $\mathrm{Al}$ contrario, i macrocambiamenti radicali e le complete sostituzioni richiederebbero uno sforzo, in termini di risorse materiali e simboliche, che solo una grande coalizione o un leader politico particolarmente carismatico sarebbero in grado di sostenere. Quanto più alta è la posta in gio- 
co, come nelle innovazioni istituzionali radicali, tanto più il conflitto politico tende ad essere elevato, le identità sociali e politiche profondamente radicate vengono minacciate, la fiducia erosa: in queste condizioni diventa ancora più difficile smantellare i vecchi ordinamenti istituzionali, e un vero cambiamento è ancora più improbabile.

Nel bricolage vengono messe insieme strutture flessibili, spesso risultato di espedienti, le quali giuocano il ruolo di «entità di riferimento» che facilitano l'interpretazione e l'azione, e la fissazione di significati e identità ${ }^{30}$. Componenti istituzionali debolmente integrate, messe insieme mediante attività di bricolage, aiutano gli attori a intraprendere azioni esplorative e a imprimere un ordine transitorio a situazioni ambigue e sfuggenti. Precisamente la loro flessibilità e la loro debole integrazione possono essere sfruttate per introdurre variazioni e reinvenzioni locali (Rice e Rogers 1980). In tal modo nuove prospettive, interessi, competenze, identità e altri elementi possono essere incorporati senza grossi attriti nell'assetto istituzionale preesistente. Nuove caratteristiche possono essere aggiunte localmente ad una configurazione esistente. Le strutture assemblate con il bricolage sono imperfette, incoerenti, frutto di espedienti, ma in qualche modo «funzionano», ed esibiscono proprietà di autocorrezione che rendono più facile una eventuale ristrutturazione ${ }^{31}$.

Se assumiamo la prospettiva del bricolage, le istituzioni assomigliano più a processi evolutivi aperti da mantenere attivi che a strutture o equilibri stabili da raggiungere una volta per tutte. Come Tocqueville ha rilevato nella sua analisi della nascente democrazia americana, $\mathrm{i}$ «complessi istituzionali» sono configurazioni poco coerenti che sono state utilizzate e «abitate» per lungo tempo in passato e che possono essere orientate a utilizzazioni future (Tocqueville 1969). Elementi, componenti, usi, significati, relazioni vengono continuamente manipolati e ricombinati per assemblare nuove configurazioni. Ordine, coerenza, e significato sono dati ex post, risultando essi più da una

30 Per «entità di riferimento» intendo un artefatto materiale o simbolico che nel corso della costruzione di una struttura o di una configurazione, e in assenza di tale struttura o configurazione, ha la proprietà di incorporare e veicolare un significato locale, e la capacità di «fissare» un ordine o coerenza locali, sulla base dei quali la struttura può successivamente svilupparsi. Su questo punto si veda Bamberger e Schon (1983).

31 E stato mostrato come queste proprietà del bricolage siano critiche per i processi di innovazione nei sistemi tecnologici e organizzativi. Vedi, per esempio, Ciborra (1992) e Lanzara (1995). 
interpretazione degli assetti emergenti che da disegni prestabiliti (Weick 1993). Questi assetti istituzionali vengono formati giustapponendo componenti locali. Essi sono soggetti a una continua attività di reinvenzione (Rice e Rogers 1980) e a fenomeni di deriva e slittamento. Mentre i componenti locali possono sempre essere modificati, l'assetto nel suo complesso ha un'elevata resilienza (Ciborra e Lanzara 1994).

Due aspetti principali devono qui essere messi in luce. Il primo è la dipendenza dalla storia. Il bricolage istituzionale è bistory-dependent, tendenzialmente retrospettivo, orientato al passato. La storia passata vincola $\mathrm{i}$ modi in cui $\mathrm{i}$ componenti vengono riassemblati e reinterpretati. In modo analogo a quanto accade in architettura, le «vestigia» del passato possono essere reimpiegate per edificare nuovi edifici istituzionali. La costruzione di istituzioni è influenzata più dalle modalità di codificazione passata che dai modi in cui si immagina il futuro. Negli assetti istituzionali preesistenti ci sono molte più risorse potenziali di quante si sia comunemente propensi a credere. Così il problema centrale del bricoleur, e della maggior parte degli imprenditori istituzionali, è in che modo trasformare vecchi elementi e risorse residuali in risorse potenziali utilizzabili per il futuro.

Il secondo aspetto è quello dell'interazione. L'effetto complessivo delle attività di bricolage sembra dipendere più dall'interazione sociale che dalla prevalenza di uno specifico stile di risposta. Nella costruzione di istituzioni una moltitudine di attori sociali e politici, che utilizzano logiche d'azione e principi di legittimazione molteplici e conflittuali, interagiscono nel corso del tempo all'interno di un'ecologia di interazioni, e l'effetto di composizione delle interazioni può eventualmente cristallizzarsi in una configurazione o assetto istituzionale. Il bricolage privilegia la logica combinatoria, i legami deboli (Weick 1976) e i processi politici a «cestino di rifiuti» (Kingdon 1984). Come principio di progettazione, il bricolage sembra essere compatibile con l'organizzazione della diversità e con l'evoluzione dei sistemi complessi. La sua logica è la «logica del disordine» (Warglien e Masuch 1996).

Si può pensare che l'attività di bricolage al margine di strutture consolidate sia null'altro che una forma di exploitation. $\mathrm{Ma}$ questa impressione è fuorviante. Il rimpasto, la trasposizione e la ricombinazione di «pezzi» di istituzioni preesistenti possono essere atti di «invenzione creativa» (Rice e Rogers 1980). Una 
sequenza di cambiamenti locali apparentemente modesti può portare a sensibili trasformazioni nelle strutture, nelle funzioni, e nei significati di un sistema complesso. Il bricolage conduce a fenomeni graduali di deriva e slittamento di una istituzione o di un codice, i quali incrementano la loro complessità strutturale senza modificare il loro nucleo di regole fondamentali. Come March ha fatto notare, le istituzioni a volte subiscono notevoli trasformazioni attraverso «cascate» di piccoli passi, e mutamenti sostanziali sono dovuti a volte agli effetti cumulativi di variazioni incrementali con carattere locale (March 1981). In altre parole il bricolage, sebbene basato su strategie modeste, può avere proprietà generative.

L'attività di bricolage dunque non è né pura exploitation né pura exploration. Piuttosto, essa combina e compone le due logiche. Da una parte sfrutta le caratteristiche e le proprietà delle strutture esistenti per favorire l'interazione e la generatività. Dall'altra permette una certa quantità di esplorazione e di variabilità in un ambiente relativamente sicuro. In tal modo il bricolage risponde ai due requisiti contraddittori della diversità $\mathrm{e}$ dell'affidabilità: esso compensa i vantaggi di una riduzione di variabilità con gli svantaggi provocati dalla mancanza di innovazioni radicali, e allo stesso tempo compensa i vantaggi di un aumento di variabilità con gli svantaggi di una riduzione di affidabilità. Mantiene le strutture abbastanza flessibili per rendere possibili eventuali ulteriori manipolazioni, ma non così debolmente integrate da vanificare ogni sforzo per produrre una qualche forma di coerenza e di stabilità locale. Gioca sull'ambiguità e sull'equivocità dei significati in modo da lasciare aperte nuove possibilità di interpretazione, ma non oltre una certa soglia al di là della quale prevarrebbero il rumore e la confusione. $\mathrm{Al}$ tempo stesso garantisce una certa «quantità» di struttura in modo da prevenire adattamenti e rimpasti sempre più frequenti e affrettati (Offe 1992). In effetti, troppo bricolage potrebbe causare non intenzionalmente un eccesso di instabilità delle regole (Zhou 1993). In tal modo una certa quantità di improvvisazione è permessa, senza incorrere nei rischi provocati dagli esiti potenzialmente distruttivi di una ristrutturazione radicale. Il bricolage è dunque espressione di una certa saggezza: se le istituzioni sono «carriers of history» (David 1992), si può dire allora che il bricolage istituzionale sia una manifestazione della saggezza della storia. 


\section{Osservazioni conclusive}

In questo saggio ho cercato di analizzare i processi autodistruttivi che rendono difficile la costruzione di istituzioni e ho suggerito alcuni modesti meccanismi che possono contrastarli. Meccanismi normali, di tutti i giorni, apparentemente innocui, dei quali gli attori non sempre sono consapevoli, possono inavvertitamente produrre, nel corso del tempo, sia esiti positivi che negativi, e tali esiti possono accadere al di là e a dispetto delle intenzioni, dei progetti, e dei desideri degli attori stessi. La costruzione di istituzioni avviene attraverso meccanismi che facilitano l'equilibrio dinamico tra strategie di ricerca ed esplorazione di nuove possibilità da un lato, e strategie di sfruttamento e perfezionamento degli assetti esistenti dall'altro, in modo da far fronte alle incoerenze e ai dilemmi nell'allocazione intertemporale delle risorse. Sia l'exploration che l'exploitation sono necessarie, ma l'eccesso dell'una o dell'altra produce risultati autodistruttivi. Sia il consolidamento dell'ordine che l'accettazione della variabilità sono aspetti importanti della costruzione di istituzioni, ma troppo ordine o troppa variabilità si volgono prima o poi in fenomeni perversi.

Ho individuato nelle incoerenze e nelle distorsioni dell'allocazione intertemporale delle risorse una fonte di difficoltà nei processi di ricostruzione istituzionale. Non è facile, anche se è possibile, apprendere a sciogliere o ad aggirare i dilemmi che la complessa dinamica dell'allocazione pone all'azione individuale e collettiva. Tali difficoltà si innestano sui ben noti problemi distributivi posti dalla complessità politica, che in questo lavoro, pur evocati di tanto in tanto, sono stati tenuti sullo sfondo dell'argomentazione. All'interrogativo posto in apertura del saggio, «perché è difficile costruire un'istituzione?» è stata dunque data una risposta parziale. $\grave{E}$ stata qui trattata solo una parte del problema. Tuttavia, io credo, una parte importante.

\section{Riferimenti bibliografici}

Abell, P. (1995), The New Institutionalism and Rational Choice Theory, in W.R. Scott e S. Christensen (a cura di), The Institutional Construction of Organizations, Thousands Oaks, Sage Publications, pp. 3-14.

Alberti, G. (1996), «Movimientismo» and Democracy: An Analytic Fra- 
mework and the Peruvian Case Study, in Eli Dinitz (a cura di), The Challenge of Democracy in Latin America, Rethinking StateSociety Relationships, Rio de Janeiro, IPSA-IUPERJ.

Argyris, C. e D.A. Schon (1996), Organizational Learning II, revised edition, Reading, Mass, Addison-Wesley.

Arthur, B.A. (1988), Self-reinforcing Mechanisms in Economics, in P.W. Anderson e K.J. Arrow (a cura di), The Economy as an Evolving Complex System, Menlo Park, Calif., Addison-Wesley, pp. 9-32.

- (1989), Competing Technologies, Increasing Returns, and Lock-in by Historical Events, in «The Economic Journal», 99 (394), marzo, pp. 116-131.

Axelrod, R. (1984), The Evolution of Cooperation, New York, Basic Books.

Baldi, B. (1996), Institutional Change Vs. Institutional Persistence? The Transformation of the U.S. Regulatory Commission since Three Mile Island, preliminary paper, Disaster Research Center, University of Delaware, gennaio.

Bamberger, J. e D.A. Schon (1983), Learning as Reflective Conversation with Materials, in «Art Education», marzo, pp. 68-73.

Ciborra, C. (1992), From Thinking to Tinkering: The Grassroots of Strategic Information Systems, in «The Information Society», 8, pp. 297-309.

Ciborra, C. e G.F. Lanzara (1994), Formative Contexts and Information Technology. Understanding the Dynamics of Innovation in Organizations, in «Accounting, Management, and Information Technology», vol. 4, pp. 61-86.

David, P. (1985), Clio and the Economics of QWERTY, in «American Economic Association Papers and Proceedings», maggio, pp. 332337.

- (1986), Understanding the Economics of QWERTY. The Necessity of History, in W. Parker (a cura di), Economic History and the Modern Historian, London, Blackwell, pp. 30-49.

- (1992), Why Institutions are the "Carriers of History». Notes on Path-dependence and the Evolution of Conventions, Organizations and Institutions, Stanford Institute for Theoretical Economics, Stanford University, mimeo, october, pp. 1-25.

Elster, J. (1984), Ulysses and the Sirens, revised edition, Cambridge, UK, Cambridge University Press (trad. it. Ulisse e le sirene. Indagini sulla razionalità e l'irrazionalità, Bologna, Il Mulino, 1983).

- (1989), The Cement of Society. A Study of Social Order, Cambridge, Cambridge University Press (trad. it. Il cemento della società. Uno studio sull'ordine sociale, Bologna, Il Mulino, 1995).

- (1993), Political Psychology, Cambridge, UK, Cambridge University Press.

Etheredge, L.S. (1976), The Case of the Unreturned Cafeteria Trays, Washington, DC, American Political Science Association. 
Gambetta, D. (a cura di) (1988a), Trust. Making and Breaking Cooperative Relationships, New York, Basil Blackwell (trad. it. Le strategie della fiducia, Torino, Einaudi, 1989).

- (1988b), Can we Trust Trust? in Gambetta (1988a).

- (1992), La mafia siciliana, Torino, Einaudi.

Garrett, G. e B.R. Weingast (1993), Ideas, Interests, and Institutions: Constructing the European Community's Internal Market, in J. Goldstein e R.O. Keohane (a cura di), Ideas and Foreign Policy. Beliefs, Institutions, and Political Change, Ithaca, NY, Cornell University Press, pp. 173-206.

Genschel, P. (1997), The Dynamics of Inertia: Institutional Persistence and Institutional Change in Telecommunications and Health Care, in «Governance» vol. 10, n. 1, pp. 43-66.

Grandori, A. (1995), Modelli di organizzazione pluralistica: il contributo europeo alla ricerca sui processi decisionali, in S.B. Bacharach, P. Gagliardi e B. Mundell (a cura di), Il pensiero organizzativo europeo, Milano, Guerini e Associati.

Granovetter, M. (1985), Economic Action and Social Structure: The Problem of Embeddedness, in «American Journal of Sociology», 91, pp. 481-510.

- (1990), The Old and the New Economic Sociology: A History and an Agenda, in R. Friedland e A.F. Robertson (a cura di), Beyond the Marketplace. Rethinking Economy and Society, New York, Aldine de Gruyter.

Guarnieri, C. (1997), Magistratura e sistema politico nella Storia d'Italia, in R. Romanelli (a cura di), Storia della Magistratura, Bologna, Il Mulino, in corso di stampa.

Jepperson, R.L. (1991), Institutions, Institutional Effects, and Institutionalism, in W.W. Powell e P.J. Dimaggio (a cura di), The New Institutionalism in Organizational Analysis, Chicago, University of Chicago Press.

Hardin, G. (1968), The Tragedy of the Commons, in «Science», 162 (13 december), pp. 1243-1248.

Kahneman, D., J.L. Knetsch e R.H. Thaler (1991), The Endowment Effect, Loss Aversion, and Status Quo Bias, in «Journal of Economic Perspectives», vol. 5, n. 1, pp. 193-206.

Kingdon, J.W. (1984), Agendas, Alternatives, and Public Policies, Boston, Little, Brown and Company.

Kreps, D. (1990), Corporate Culture and Economic Theory, in J. Alt e $\mathrm{K}$. Shepsle (a cura di), Perspectives on Positive Political Economy, New York, Cambridge University Press.

Kuran, T. (1988), The Tenacious Past: Theories of Personal and Collective Conservatism, in «Journal of Economic Behavior and Organization», 10, pp. 143-171.

Lant, T.K. (1992), Aspiration Level Adaptation: An Empirical Exploration, in «Management Science», 38, pp. 311-328. 
Lanzara, G.F. (1995), Tecnologia, sensemaking, e contesti formativi. Gli esperimenti di videoregistrazione del processo penale, in «Rivista Trimestrale di Scienza dell'Amministrazione», 1, pp. 77-109.

Levinthal, D.A. e J.G. March (1993), The Myopia of Learning, in «Strategic Management Journal», 14, pp. 95-112.

Levi-Strauss, C. (1962), La pensée sauvage, Paris, Seuil (trad. it. Il pensiero selvaggio, Milano, Il Saggiatore, 1964).

Levitt, B. e J.G. March (1988), Organizational Learning, in «Annual Review of Sociology», 1988, 14, pp. 319-340.

Lijparht, A. (1984), Democracies: Patterns of Majoritarian and Consensus Government in 21 Countries, New Haven, Yale University Press (trad. it. Le democrazie contemporanee, Bologna, Il Mulino, 1988).

Luhmann, N. (1979), Trust and Power, Chichester, UK, John Wiley \& Sons.

March, J.G. (1981), Footnotes to Organizational Change, in «Administrative Science Quarterly», 26, pp. 563-577.

- (1988), Variable Risk Preferences and Adaptive Aspirations, in «Journal of Economic Behavior and Organization», 9, pp. 5-24.

- (1991), Exploration and Exploitation in Organizational Learning, in «Organization Science», 2, 1, pp. 71-87.

March, J.G. e J.P. Olsen (1984), The New Institutionalism: Organizational Factors in Political Life, in «American Political Science Review», 78, pp. 734-749.

- (1989), Rediscovering Institutions. The Organizational Basis of Politics, New York, The Free Press (trad. it. Riscoprire le istituzioni, Bologna, Il Mulino, 1992).

North, D.C. (1990), Institutions, Institutional Change and Economic Performance, Cambridge, UK, Cambridge University Press (trad. it. Istituzioni, cambiamento istituzionale, evoluzione dell'economia, Bologna, Il Mulino, 1994).

Offe, C. (1992), Designing Institutions for East European Transitions, draft paper presented for the «Institutional Design Conference», The Australian National University, Research School of Social Science, 7-8 dicembre.

Olsen, J.P. (1992), Analysing Institutional Dynamics, in «Staatswissenschaften und Staatspraxis», Heft 2, 3. Jahrgang, pp. 247-271.

Olson, M. (1966), The Logic of Collective Action, Cambridge, Harvard University Press (trad. it. La logica dell'azione collettiva, Milano, Feltrinelli, 1983).

Orren, K. e S. Skowronek (1991), Beyond the Iconography of Order: Notes for a «New Institutionalism», in L.C. Dodd e C. Jillson (a cura di), The Dynamics of American Politics, Boulder, Westview Press, pp. 311-330.

Pagden, A. (1988), La distruzione della fiducia e le conseguenze economiche a Napoli nel XVIII secolo, in Gambetta (1988a), pp. 165-182. 
Pizzorno, A. (1986a), Sul confronto intertemporale delle utilità, in «Stato e Mercato», 16, pp. 3-25.

- (1986b), Some Other Kind of Otherness: a Critique of «Rational Choice» Theories, in A. Foxley, M. McPherson, G. O'Donnell (a cura di), Development, Democracy and the Art of Trespassing. Essays in Honor of Albert Hirschman, Notre Dame, University of Notre Dame Press.

- (1995), Note sull'uso della nozione di sé nell'indagine sociale, manoscritto non pubblicato, Istituto Universitario Europeo, San Domenico di Fiesole, pp. 1-23.

Poni, C. (1995), Standards, Trust, and Civil Society: Measuring the Thickness of Quality Silk Thread, saggio presentato al Seminario di ricerca su «Istituzioni e Institution Building», Dipartimento di Organizzazione e Sistema Politico, Università di Bologna, 4 dicembre.

Putnam, R.D. (1993), Making Democracy Work. Civic Traditions in Modern Italy, Princeton, Princeton University Press (trad. it. La tradizione civica nelle regioni italiane, Milano, Mondadori, 1993).

Rice, R. e E. Rogers (1980), Reinvention in the Innovation Process, in «Knowledge», I, 4, pp. 488-514.

Sartori, G. (1994), Comparative Constitutional Engineering: An Inquiry into Structures, Incentives and Outcomes, New York, Mcmillan Press (trad. it. Ingegneria istituzionale comparata, Bologna, Il Mulino, 1995).

Scazzieri, R. (1994), Sulla teoria economica della società civile, saggio presentato all'Accademia delle Scienze dell'Istituto di Bologna, Classe di Scienze Morali, Bologna, 4 febbraio.

Schelling, T.C. (1978), Micromotives and Macrobebavior, New York, W.W. Norton and Company.

- (1980), The Strategy of Conflict, revised edition, Cambridge, Harvard University Press (prima edizione 1964).

Skowronek, S. (1982), Building a New American State. The Expansion of National Administrative Capacities 1877-1920, Cambridge, UK, Cambridge University Press.

Stark, D. (1994), Capitalismo su progetto? Nuove combinazioni tra pubblico e privato nell'Europa dell'Est, in «Stato e Mercato», 42, pp. 303-321.

- (1996), Recombinant Property in East European Capitalism, in «American Journal of Sociology», vol. 101, gennaio.

Stinchcombe, A.L. (1968), Constructing Social Theories, New York, Harcourt, Brace \& World.

Sztompka, P. (1996), La fiducia nelle società post-comuniste. Una risorsa scomparsa, Soveria Mannelli, Rubbettino.

Taylor, M. (1987), The Possibility of Cooperation, Cambridge, Cambridge University Press.

Tomasi di Lampedusa, G. (1958), Il gattopardo, Milano, Feltrinelli. 
Tocqueville, A. de (1969), Democracy in America, a cura di J.P. Mayer, tradotto da George Lawrence, Garden City, Anchor Books.

Toynbee, A. (1934-1959), A Study of History, London.

Unger, R.M. (1987), False Necessity, Cambridge, Cambridge University Press.

Warglien, M. e M. Masuch (a cura di), (1996), The Logic of Organizational Disorder, Berlino-New York, Walter de Gruyter.

Weick, K.E. (1976), Educational Organizations as Loosely Coupled Systems, in «Administrative Science Quarterly», 21, pp. 1-19.

- (1993), Organizational Redesign as Improvisation, in G.P. Huber e W.H. Glick (a cura di), Organizational Change and Redesign, New York, Oxford University Press, pp. 346-379.

- (1995), Sensemaking in Organizations, Thousands Oaks, Sage Publications.

Zhou, X. (1993), The Dynamics of Organizational Rules, in «American Journal of Sociology», 98, 5, pp. 1134-1166. 